\title{
Catalytic Asymmetric Carbohydroxylation of Alkenes By Single-Pot Diboration/Suzuki Cross-Coupling/Oxidation Reaction.
}

\author{
Steven P. Miller, Jeremy B. Morgan, Felix J. Nepveux V and James P. Morken* \\ Department of Chemistry, Venable and Kenan Laboratories
}

University of North Carolina, Chapel Hill, NC 27599-3290

\section{Supplementary Material}

\begin{abstract}
General. ${ }^{1} \mathrm{H}$ NMR spectra were recorded on Bruker DRX (400 MHz or 300 $\mathrm{MHz}$. Chemical shifts are reported in ppm from tetramethylsilane with the solvent resonance as the internal standard $\left(\mathrm{CDCl}_{3}: 7.24 \mathrm{ppm}\right)$. Data are reported as follows: chemical shift, integration, multiplicity $(\mathrm{s}=$ singlet, $\mathrm{d}=$ doublet, $\mathrm{t}=$ triplet, $\mathrm{q}=$ quartet, $\mathrm{br}$ $=$ broad, $\mathrm{m}=$ multiplet), coupling constants $(\mathrm{Hz})$ and assignment. ${ }^{13} \mathrm{C}$ NMR were recorded on a Bruker DRX $400(100 \mathrm{MHz})$ spectrometer with complete proton decoupling. Chemical shifts are reported in ppm from tetramethylsilane with the solvent as the internal standard $\left(\mathrm{CDCl}_{3}: 77.0 \mathrm{ppm}\right)$. High resolution mass spectrometry was performed by the University of Minnesota Mass Spectrometry Service Laboratory. Infrared (IR) spectra were obtained using a ASI ReactIR 1000.
\end{abstract}

Liquid chromatography was performed using forced flow (flash chromatography) on silica gel $\left(\mathrm{SiO}_{2}, 32\right.$ to $\left.63 \mu \mathrm{m}\right)$ purchased from Scientific Adsorbents, Inc. Thin layer chromatography (TLC) was performed on EM science $0.25 \mathrm{~mm}$ silica gel 60 plates. Visualization was achieved UV light or phosphomolybdic acid in ethanol followed by heating.

Analytical gas-liquid chromatography (GLC) was performed on a HewlettPackard 6890 Series chromatograph equipped with a CTC Analysis Combi Pal autosampler by Leap Technologies (Carrboro, NC), a split mode capillary injection system, a flame ionization detector and a Supelco $\beta$-dex 120 column with helium as the carrier gas. Analytical high performance liquid chromatography (HPLC) was performed on a Shimadzu liquid chromatograph equipped with a UV detector and a Chiralcel OD column. Analytical supercritical fluid chromatography (SFC) was performed on a Berger Instruments supercritical fluid chromatograph equipped with an Alcott autosampler and a Knauer UV detector. Microwave promoted reactions were performed with a CEM Corporation Discover reaction microwave equipped with a pressure sensing device.

All reactions were conducted in oven and flame dried glassware under an inert atmosphere of argon. Alkene starting materials were commercially available, except for (2,2-Dimethyl-but-3-enyloxymethyl)-benzene which was generated by a methylene 
Wittig reaction on the corresponding aldehyde. [(nbd) $\mathrm{Rh}(\mathrm{acac})]$ was generated from $[(\mathrm{nbd}) \mathrm{RhCl}]_{2}$ by reaction with sodium acetylacetone in toluene at $40^{\circ} \mathrm{C}$ (Burke, J. M.; Coapes, R.B.; Goeta, A.E.; Howard, J.A.K.; Marder, T.B.; Robins, E.G.; Westcott, S.A. J. Organomet. Chem. 2002, 649, 199.) Bis(catecholato)diboron, (dppf) $\mathrm{PdCl}_{2} \cdot \mathrm{CH}_{2} \mathrm{Cl}_{2}$, cesium carbonate, anhydrous $\mathrm{THF}, \mathrm{Pd}(\mathrm{OAc})_{2}$, and all coupling partners were purchased from Aldrich Chemical Company. Both enantiomers of Quinap were purchased from Strem Chemical Company or Acros Chemical Company. 2-(Di- tbutylphosphino)biphenyl was purchased from Strem Chemical Company. All reagents were used as received.

\section{Representative Procedure for Catalytic, Enantioselective Diboration Reaction (Table 1).}

An oven-dried $20 \mathrm{~mL}$ vial equipped with a stir-bar was charged with $5.8 \mathrm{mg}$ $(0.020 \mathrm{mmol})$ of (bicyclo[2.2.1]hepta-2,5-diene)-(2,4-pentanedionato)-rhodium(I), 8.7 $\mathrm{mg}(0.020 \mathrm{mmol})$ of $(S)$-Quinap, and $1.6 \mathrm{~mL}$ of THF under an inert atmosphere of argon in a dry-box. The resultant yellow solution was stirred for 5 minutes. After this time, $141 \mathrm{mg}(0.59 \mathrm{mmol})$ of bis(catecholato)diboron was added to the solution under argon. The solution turned immediately from yellow to dark brownish-red. The solution was allowed to stir for 5 minutes. After this time, $50 \mathrm{mg}(0.39 \mathrm{mmol})$ of 3,3-dimethyl-1heptene was added to the solution under argon. The vial was sealed with a screw-cap and removed from the dry box, where the solution was allowed to stir for 6 hours at ambient temperature. After this time, $1.25 \mathrm{~mL}$ of $3 \mathrm{M} \mathrm{NaOH}$ and then $0.800 \mathrm{~mL}$ of $30 \% \mathrm{H}_{2} \mathrm{O}_{2}$ (dropwise with caution) were added under nitrogen. The solution was allowed to stir at ambient temperature for 6 hours. The solution was then quenched with $2 \mathrm{~mL}$ of saturated aqueous $\mathrm{Na}_{2} \mathrm{~S}_{2} \mathrm{O}_{3}$ and $10 \mathrm{~mL}$ of $1 \mathrm{M} \mathrm{NaOH}$. The mixture was extracted with ethyl acetate $(3 \times 25 \mathrm{~mL})$ and the combined organic layers were washed with brine $(1 \times 10$ $\mathrm{mL}$ ). The organic layers were then dried over anhydrous $\mathrm{MgSO}_{4}$, filtered, and the solvent removed by rotary evaporation. The crude material was purified by silica gel chromatography $(2: 1$ hexanes : ethyl acetate) to provide $52 \mathrm{mg}(82 \%)$ of pure $(R)-3,3-$ dimethyl-heptane-1,2-diol.

\section{Representative Procedure for Catalytic, Enantioselective Diboration/Suzuki Cross- Coupling/Oxidation Reaction (Table 2).}

An oven-dried $20 \mathrm{~mL}$ vial equipped with a stir-bar was charged with $3.7 \mathrm{mg}$ $(0.013 \mathrm{mmol})$ of (bicyclo[2.2.1]hepta-2,5-diene)-(2,4-pentanedionato)-rhodium(I), 5.5 $\mathrm{mg}(0.013 \mathrm{mmol})$ of $(S)$-Quinap, and $1.0 \mathrm{~mL}$ of THF under an inert atmosphere of argon in a dry-box. The resultant yellow solution was stirred for 5 minutes. After this time, 89 $\mathrm{mg}(0.38 \mathrm{mmol})$ of bis(catecholato)diboron was added to the solution under argon. The solution turned immediately from yellow to dark brownish-red. The solution was allowed to stir for 5 minutes. After this time, $34 \mu \mathrm{L}(0.25 \mathrm{mmol})$ of 3,3-dimethyl-1butene was transferred to the solution under argon. The vial was sealed with a screw-cap and removed from the dry box, where the solution was allowed to stir for 6 hours at ambient temperature. After this time, the reaction mixture was diluted with $1.5 \mathrm{~mL}$ of 
THF. To the reaction flask was weighed $20 \mathrm{mg}(0.025 \mathrm{mmol})$ of [1,1'bis(diphenylphosphino)ferrocene]dichloropalladium(II) $\cdot \mathrm{CH}_{2} \mathrm{Cl}_{2}, 244 \mathrm{mg}(0.75 \mathrm{mmol})$ of cesium carbonate, and $94 \mathrm{mg}(0.5 \mathrm{mmol})$ of 3-bromoanisole. The resulting suspension was stirred for 30 seconds, and $250 \mu \mathrm{L}$ of deoxygenated water was added. The reaction vial was sealed and heated to $80^{\circ} \mathrm{C}$ for 18 hours. After this time, $0.800 \mathrm{~mL}$ of $3 \mathrm{M} \mathrm{NaOH}$ was added, and the mixture was cooled to $0^{\circ} \mathrm{C}$. Under nitrogen, $0.500 \mathrm{~mL}$ of $30 \% \mathrm{H}_{2} \mathrm{O}_{2}$ was transferred (dropwise with caution). The solution was allowed to stir at ambient temperature for 6 hours. The solution was then quenched with $2 \mathrm{~mL}$ of saturated aqueous $\mathrm{Na}_{2} \mathrm{~S}_{2} \mathrm{O}_{3}$ and $10 \mathrm{~mL}$ of $1 \mathrm{M} \mathrm{NaOH}$. The mixture was extracted with ethyl acetate $(3 \times 25$ $\mathrm{mL})$ and the combined organic layers were washed with brine $(1 \times 10 \mathrm{~mL})$. The organic layers were then dried over anhydrous $\mathrm{MgSO}_{4}$, filtered, and the solvent removed by rotary evaporation. The crude material was purified by silica gel chromatography $(8: 1$ hexanes : ethyl acetate) to provide $40 \mathrm{mg}$ (77\%) of pure (S)-1-(3-methoxy-phenyl)-3,3dimethyl-butan-2-ol.

\section{Representative Procedure for Catalytic, Enantioselective Diboration/Microwave Suzuki Cross-Coupling/Oxidation Reaction (Scheme 2).}

Diboration was conducted using the standard procedure listed above. To the microwave reaction vessel was weighed $20 \mathrm{mg}(0.025 \mathrm{mmol})$ of $[1,1$ 'bis(diphenylphosphino)ferrocene]dichloropalladium(II) $\cdot \mathrm{CH}_{2} \mathrm{Cl}_{2}$ and $244 \mathrm{mg}(0.75 \mathrm{mmol})$ of cesium carbonate. The diboration solution was diluted with $1.5 \mathrm{~mL}$ of dry THF and transferred to the microwave reaction vessel. Phenyl triflate $(113 \mathrm{mg}, 0.5 \mathrm{mmol})$ was weighed into the vessel and the suspension was stirred briefly. The vessel was sealed under argon. Microwave activation was conducted for 1 hour at $50 \mathrm{~W}$ and $80^{\circ} \mathrm{C}$. The mixture was cooled to room temperature and subjected to the same oxidation and isolation condition as reported above. The crude material was purified by silica gel chromatography $(10: 1$ hexanes : ethyl acetate) to provide $31 \mathrm{mg}(70 \%)$ of pure $(R)-3,3-$ dimethyl-1-phenyl-butan-2-ol.

\section{Procedure for Etherification Reaction (Scheme 3).}

An oven-dried $5 \mathrm{~mL}$ vial equipped with a stir-bar was charged with $38 \mathrm{mg}(0.125$ mmol) of (R)-1-(2-Chloro-phenyl)-3,3-dimethyl-4- $p$-tolyl-butan-2-ol, $2.8 \mathrm{mg}(0.0125$ mmol) of palladium (II) acetate, $4.5 \mathrm{mg} \quad(0.015 \mathrm{mmol})$ of (2-(Di-tbutylphosphino)biphenyl, $61 \mathrm{mg}(0.188 \mathrm{mmol})$ of $\mathrm{Cs}_{2} \mathrm{CO}_{3}$, and $0.25 \mathrm{~mL}$ of THF under an inert atmosphere of argon in a dry-box. The vial was sealed with a screw-cap and removed from the dry box. The resultant orange solution was placed in an $80^{\circ} \mathrm{C}$ oil bath whereupon the mixture slowly turned dark brown over approximately $5 \mathrm{~min}$. The solution was stirred for 28 hours. After cooling to room temperature, the mixture was diluted with pentane, filtered through a celite pad, and concentrated to yield a dark orange oil. The crude material was purified by silica gel chromatography $(20: 1$ hexanes : ethyl acetate) to provide $32 \mathrm{mg}(96 \%)$ of pure (R)-2-(1,1-Dimethyl-2-p-tolyl-ethyl)-2,3dihydro-benzofuran. 
$\mathrm{OH} \quad(\boldsymbol{R})$-1-Cyclohexyl-ethane-1,2-diol. IR (neat): 3452 (br, s) $\mathrm{cm}^{-1} ;{ }^{1} \mathrm{H}$ $\mathrm{OH}$ NMR: $\delta 3.68(1 \mathrm{H}, \mathrm{d}, \mathrm{J}=9.6 \mathrm{~Hz}, \mathrm{CHCHOH}), 3.51\left(1 \mathrm{H}, \mathrm{m}, \mathrm{CH}_{2} \mathrm{OH}\right)$, $3.42\left(1 \mathrm{H}, \mathrm{m}, \mathrm{CH}_{2} \mathrm{OH}\right), 2.18(2 \mathrm{H}$, broad s, OH $), 1.84(1 \mathrm{H}, \mathrm{d}, \mathrm{J}=12.4$ $\left.\mathrm{Hz}, \mathrm{C}_{6} \mathbf{H}_{11}\right), 1.73\left(2 \mathrm{H}, \mathrm{m}, \mathrm{C}_{6} \mathbf{H}_{11}\right), 1.63\left(2 \mathrm{H}, \mathrm{m}, \mathrm{C}_{6} \mathbf{H}_{11}\right), 0.9-1.5(6 \mathrm{H}, \mathrm{m}$, $\mathrm{C}_{6} \mathbf{H}_{11}$ ); ${ }^{13} \mathrm{C}$ NMR: $\delta$ 76.5, 64.8, 40.7, 28.9, 28.6, 26.3, 26.0, 25.9. HRMS (FAB) Calc'd for $\mathrm{C}_{8} \mathrm{H}_{16} \mathrm{O}_{2}(\mathrm{M}+\mathrm{Na})^{+}:$167.1043. Found $(\mathrm{M}+\mathrm{Na})^{+}: 167.1044$.

Proof of Stereochemistry. Stereochemical ratios were determined in comparison to authentic racemic materials prepared by osmium tetraoxide catalyzed dihydroxylation. Absolute stereochemistry established in comparison to authentic $(R)$ isomer prepared via a Sharpless asymmetric dihydroxylation (Becker, H.; King, S. B.; Taniguchi, M.; Vanhessche, K.; Sharpless, K. B. J. Org. Chem. 1995, 60, 3940).

Chiral GLC ( $\beta$-dex, Supelco, $\left.120^{\circ} \mathrm{C}\right)$ analysis of diol product:

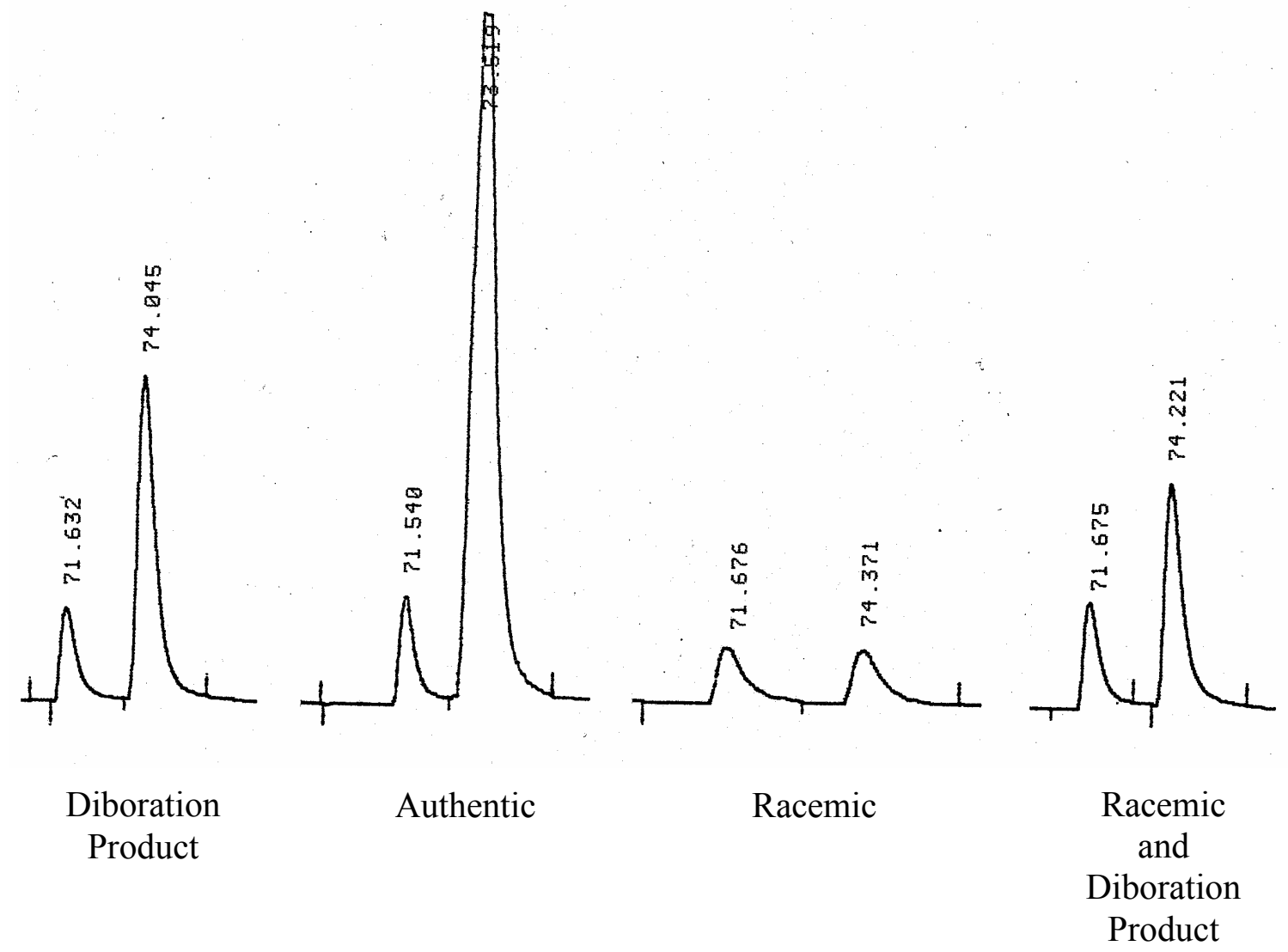


$\mathrm{OH}$
$\mathrm{C}_{8} \mathrm{H}_{17}$ $3.41\left(1 \mathrm{H}, \mathrm{dd}, \mathrm{J}=10.8 \mathrm{~Hz}, 7.6 \mathrm{~Hz}, \mathrm{CH}_{\mathrm{a}} \mathbf{H}_{\mathrm{b}} \mathrm{OH}\right), 2.06(2 \mathrm{H}$, br s, OH), 1.5-

$1.35\left(3 \mathrm{H}, \mathrm{m},-\mathbf{C H}_{2^{-}}\right), 1.35-1.15\left(11 \mathrm{H}, \mathrm{m},-\mathrm{CH}_{2^{-}}\right), 0.86\left(3 \mathrm{H}, \mathrm{t}, \mathrm{J}=7.0 \mathrm{~Hz}, \mathrm{CH}_{3}\right) ;{ }^{13} \mathrm{C}$ NMR: $\delta 72.3,66.8,33.2,31.8,29.6,29.5,29.2,25.5,22.6,14.1$. HRMS (FAB) Calc'd for $\mathrm{C}_{10} \mathrm{H}_{22} \mathrm{O}_{2}(\mathrm{M}+\mathrm{Na})^{+}:$197.1512. Found $(\mathrm{M}+\mathrm{Na})^{+}: 197.1509$.

Proof of Stereochemistry. Stereochemical ratios were determined in comparison to authentic racemic materials prepared by osmium tetraoxide catalyzed dihydroxylation. Absolute stereochemistry established in comparison to authentic $(R)$ isomer prepared via a Sharpless asymmetric dihydroxylation (Becker, H.; King, S. B.; Taniguchi, M.; Vanhessche, K.; Sharpless, K. B. J. Org. Chem. 1995, 60, 3940).

Chiral GLC ( $\beta$-dex, Supelco, $\left.120^{\circ} \mathrm{C}\right)$ analysis of diol product:

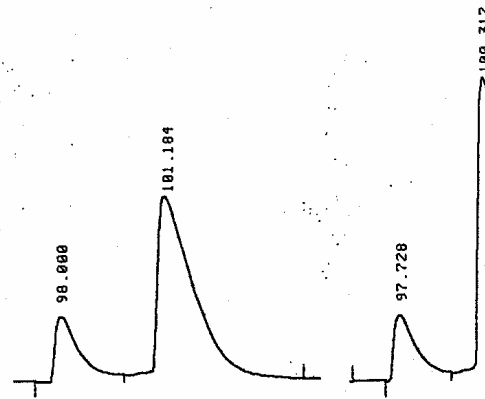

Diboration Product

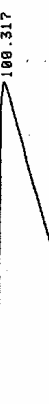

Authentic
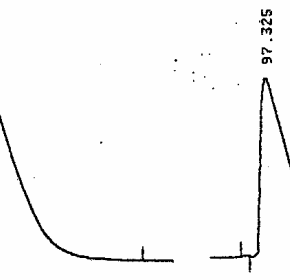

Racemic
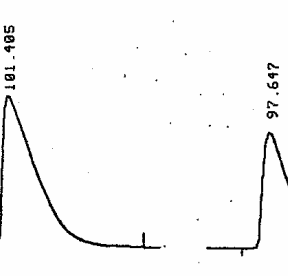

(1)

Racemic
and

Diboration

Product

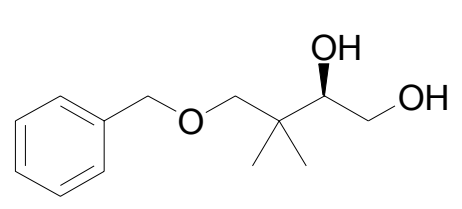

(R)-4-Benzyloxy-3,3-dimethyl-butane-1,2-diol. IR (neat): 3467 (br, s) $\mathrm{cm}^{-1} ;{ }^{1} \mathrm{H}$ NMR: $\delta$ 7.36-7.25 $(5 \mathrm{H}, \mathrm{m}$, aromatic), $4.51\left(1 \mathrm{H}, \mathrm{d}, \mathrm{J}=11.8 \mathrm{~Hz}, \mathrm{CH}_{\mathrm{a}} \mathrm{H}_{\mathrm{b}} \mathrm{Ph}\right), 4.48(1 \mathrm{H}, \mathrm{d}, \mathrm{J}=11.8$

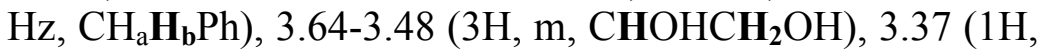
br s, OH), $3.32\left(1 \mathrm{H}, \mathrm{d}, \mathrm{J}=20.8 \mathrm{~Hz}, \mathrm{OCH}_{\mathrm{a}} \mathrm{H}_{\mathrm{b}} \mathrm{C}\right), 3.29(1 \mathrm{H}, \mathrm{d}$, $\left.\mathrm{J}=20.8 \mathrm{~Hz}, \mathrm{OCH}_{\mathrm{a}} \mathbf{H}_{\mathbf{b}} \mathrm{C}\right), 2.68(1 \mathrm{H}, \mathrm{br} \mathrm{s}, \mathrm{OH}), 0.94\left(3 \mathrm{H}, \mathrm{s}, \mathrm{CCH}_{3}\right), 0.92\left(3 \mathrm{H}, \mathrm{s}, \mathrm{CCH}_{3}\right)$; ${ }^{13} \mathrm{C}$ NMR: $\delta 137.5,128.5,127.9,127.6,78.4,78.3,73.6,62.9,37.4,23.0,20.6$. HRMS (FAB) Calc'd for $\mathrm{C}_{13} \mathrm{H}_{20} \mathrm{O}_{3}(\mathrm{M}+\mathrm{Na})^{+}: 247.1305$ Found $(\mathrm{M}+\mathrm{Na})^{+}: 247.1303$.

Proof of Stereochemistry. Stereochemical ratios could not be accurately determined for this diol product; however, selectivity can be inferred from the corresponding carbohydroxylation product. 
OH (R)-3,3-Dimethyl-butane-1,2-diol. $\quad$ IR (neat): 3438 (br, s) cm ${ }^{-1} ;{ }^{1} \mathrm{H}$ $\mathrm{OH}$ NMR: $\delta 3.73(1 \mathrm{H}, \mathrm{dd}, \mathrm{J}=10.8 \mathrm{~Hz}, 2.8 \mathrm{~Hz}, \mathrm{CHOH}), 3.47(1 \mathrm{H}, \mathrm{dd}, \mathrm{J}=10.8$ $\left.\mathrm{Hz}, 9.2 \mathrm{~Hz}, \mathrm{CH}_{\mathbf{a}} \mathrm{H}_{\mathrm{b}} \mathrm{OH}\right), 3.37\left(1 \mathrm{H}, \mathrm{dd}, \mathrm{J}=9.2 \mathrm{~Hz}, 2.8 \mathrm{~Hz}, \mathrm{CH}_{\mathrm{a}} \mathbf{H}_{\mathbf{b}} \mathrm{OH}\right)$, $2.14\left(1 \mathrm{H}\right.$, br s, OH), $1.93(1 \mathrm{H}$, br s, OH $), 0.91\left(9 \mathrm{H}, \mathrm{s}, \mathrm{C}\left(\mathrm{CH}_{3}\right)_{3}\right) ;{ }^{13} \mathrm{C} \mathrm{NMR}: \delta 79.7,63.2$, 33.5, 25.8. HRMS (FAB) Calc'd for $\mathrm{C}_{6} \mathrm{H}_{14} \mathrm{O}_{2}(\mathrm{M}+\mathrm{Na})^{+}: 141.0886$ Found $(\mathrm{M}+\mathrm{Na})^{+}$: 141.0885 .

Proof of Stereochemistry. Stereochemical ratios were determined in comparison to authentic racemic materials prepared by osmium tetraoxide catalyzed dihydroxylation. Absolute stereochemistry established in comparison to authentic $(R)$ isomer prepared via a Sharpless asymmetric dihydroxylation (Becker, H.; King, S. B.; Taniguchi, M.; Vanhessche, K.; Sharpless, K. B. J. Org. Chem. 1995, 60, 3940).

Chiral GLC ( $\beta$-dex, Supelco, $\left.120^{\circ} \mathrm{C}\right)$ analysis of diol product:

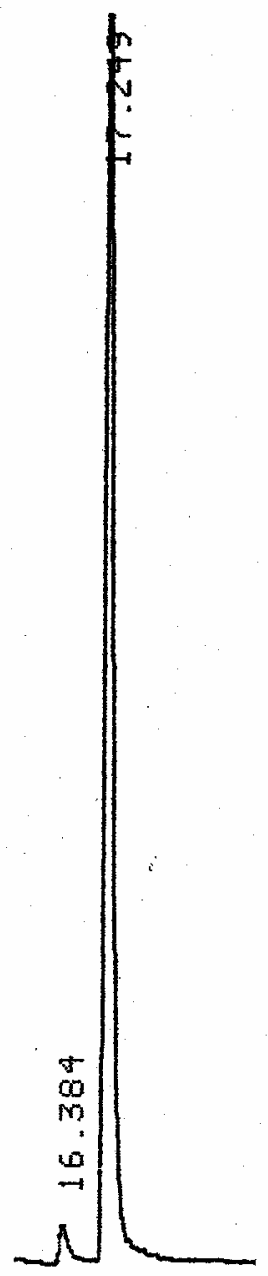

Diboration

Product

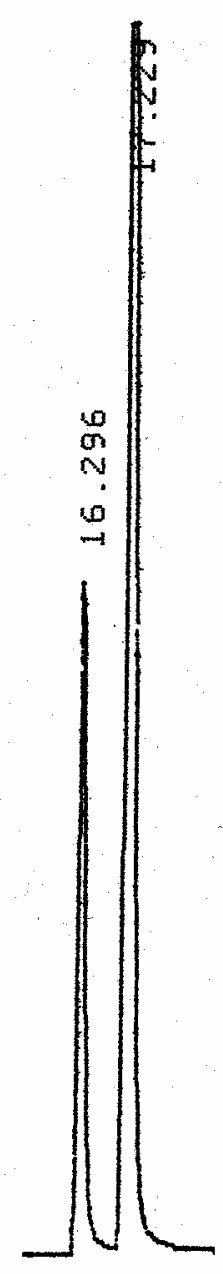

Authentic

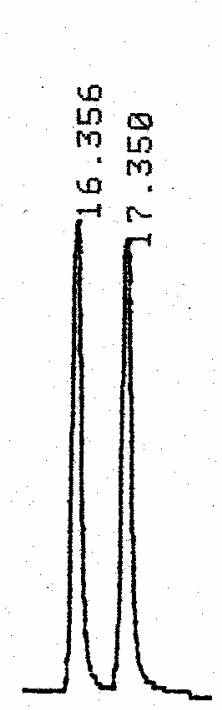

Racemic

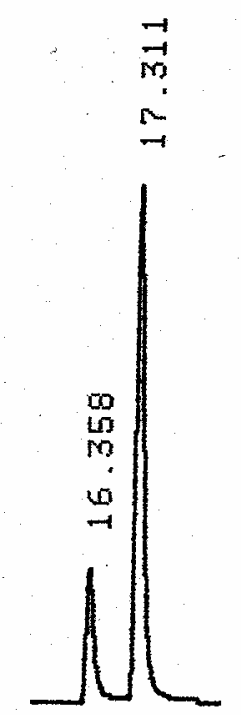

Racemic and

Diboration

Product 
<smiles>OC[C@@H](O)c1ccccc1</smiles>

(1R)-phenyl-ethane-1, 2-diol. IR (neat): 3369 (br, s), 2925 (m), 1453 (s) $\mathrm{cm}^{-1} ;{ }^{1} \mathrm{H}$ NMR: $\delta 7.41-7.26(5 \mathrm{H}, \mathrm{m}, \mathrm{Ph}), 4.81(1 \mathrm{H}, \mathrm{dd}, \mathrm{J}=8.0 \mathrm{~Hz}$, $3.6 \mathrm{~Hz}, \mathrm{PhCHOH}), 3.75\left(1 \mathrm{H}, \mathrm{dd}, \mathrm{J}=10.8 \mathrm{~Hz}, 3.6 \mathrm{~Hz}, \mathrm{CH}_{\mathrm{a}} \mathrm{H}_{\mathrm{b}} \mathrm{OH}\right), 3.65$ $\left(1 \mathrm{H}, \mathrm{dd}, \mathrm{J}=10.8 \mathrm{~Hz}, 8.0 \mathrm{~Hz}, \mathrm{CH}_{\mathrm{a}} \mathbf{H}_{\mathrm{b}} \mathrm{OH}\right), 2.25(2 \mathrm{H}$, br s, OH $) ;{ }^{13} \mathrm{C}$ NMR: $\delta 140.5,128.6,128.0,126.1,74.7,68.1$. HRMS (FAB) Calc'd for $\mathrm{C}_{8} \mathrm{H}_{10} \mathrm{O}_{2}(\mathrm{M}+$ $\left.\mathrm{NH}_{4}\right)^{+}: 156.1025$ Found $\left(\mathrm{M}+\mathrm{NH}_{4}\right)^{+}: 156.1026$.

Proof of Stereochemistry. Stereochemical ratios were determined in comparison to authentic racemic materials prepared by osmium tetraoxide catalyzed dihydroxylation. Absolute stereochemistry established in comparison to authentic $(R)$ isomer prepared via a Sharpless asymmetric dihydroxylation (Becker, H.; King, S. B.; Taniguchi, M.; Vanhessche, K.; Sharpless, K. B. J. Org. Chem. 1995, 60, 3940).

Chiral GLC ( $\beta$-dex, Supelco, $\left.130^{\circ} \mathrm{C}\right)$ analysis of diol product:

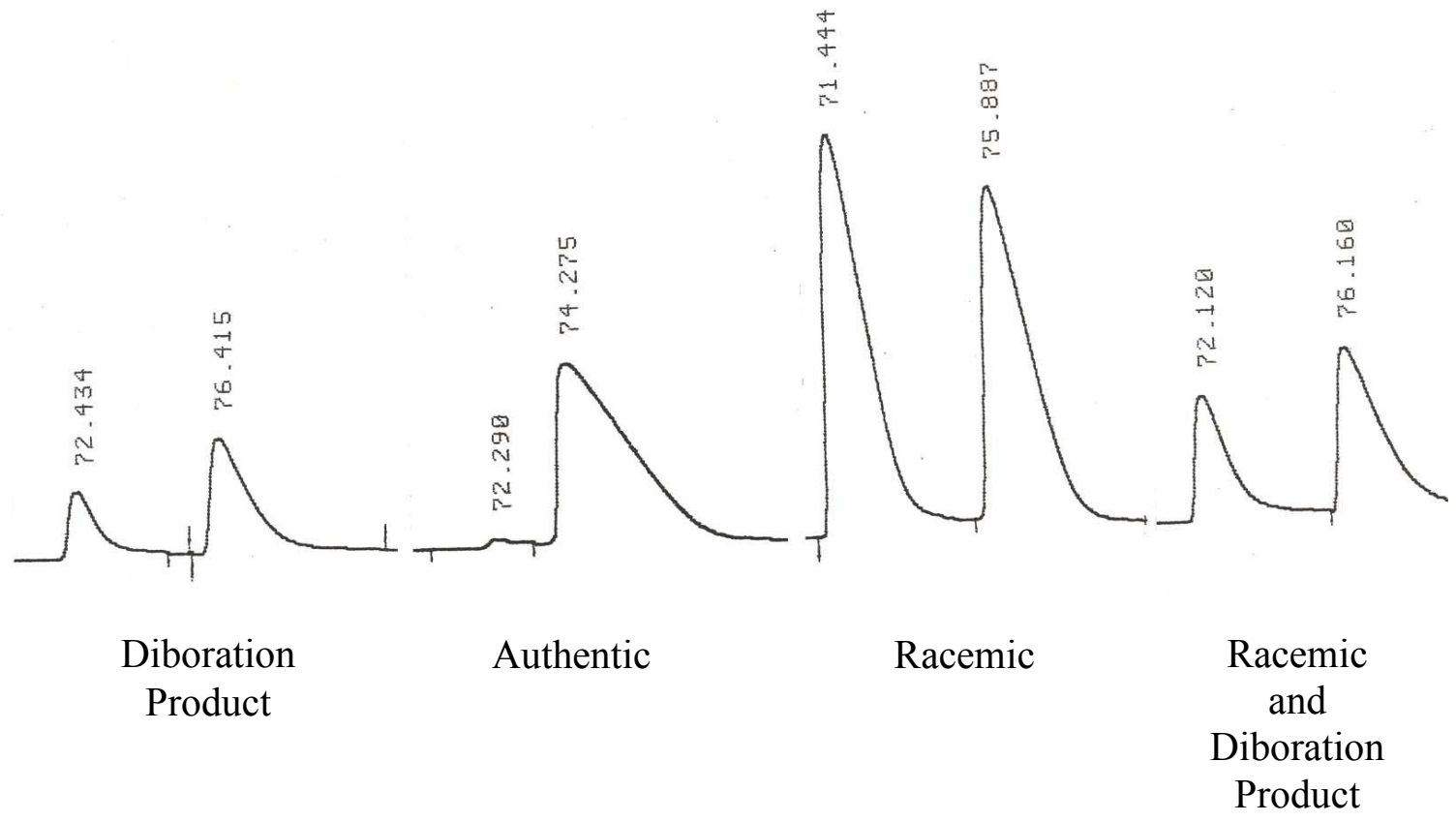




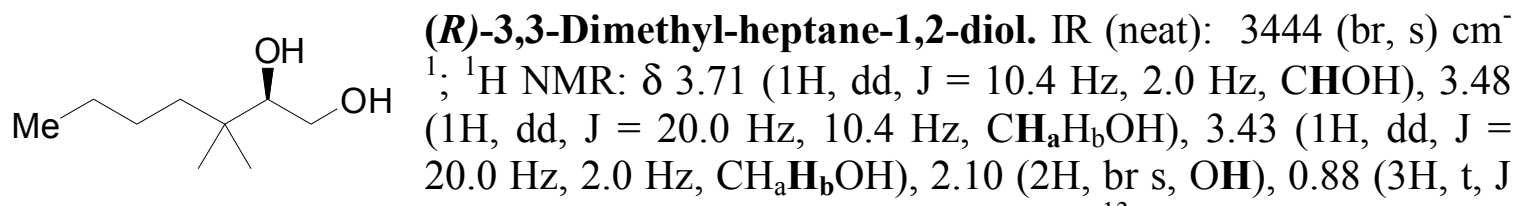
$\left.=7.0 \mathrm{~Hz}, \mathrm{CH}_{2} \mathrm{CH}_{3}\right), 0.87\left(3 \mathrm{H}, \mathrm{s}, \mathrm{CCH}_{3}\right), 0.84\left(3 \mathrm{H}, \mathrm{s}, \mathrm{CCH}_{3}\right) ;{ }^{13} \mathrm{C} \mathrm{NMR}: \delta$ 78.6, 63.0, 38.9, 36.0, 25.8, 23.6, 26.1, 22.9, 14.1. HRMS (FAB) Calc'd for $\mathrm{C}_{9} \mathrm{H}_{20} \mathrm{O}_{2}(\mathrm{M}+\mathrm{Na})^{+}$: 183.1356. Found $(\mathrm{M}+\mathrm{Na})^{+}: 183.1356$.

Proof of Stereochemistry. Stereochemical ratios were determined in comparison to authentic racemic materials prepared by osmium tetraoxide catalyzed dihydroxylation. Absolute stereochemistry established in comparison to authentic $(R)$ isomer (assignment made based on Sharpless stereochemical model) prepared via a Sharpless asymmetric dihydroxylation.

Chiral GLC ( $\beta$-dex, Supelco, $\left.110^{\circ} \mathrm{C}\right)$ analysis of diol product:

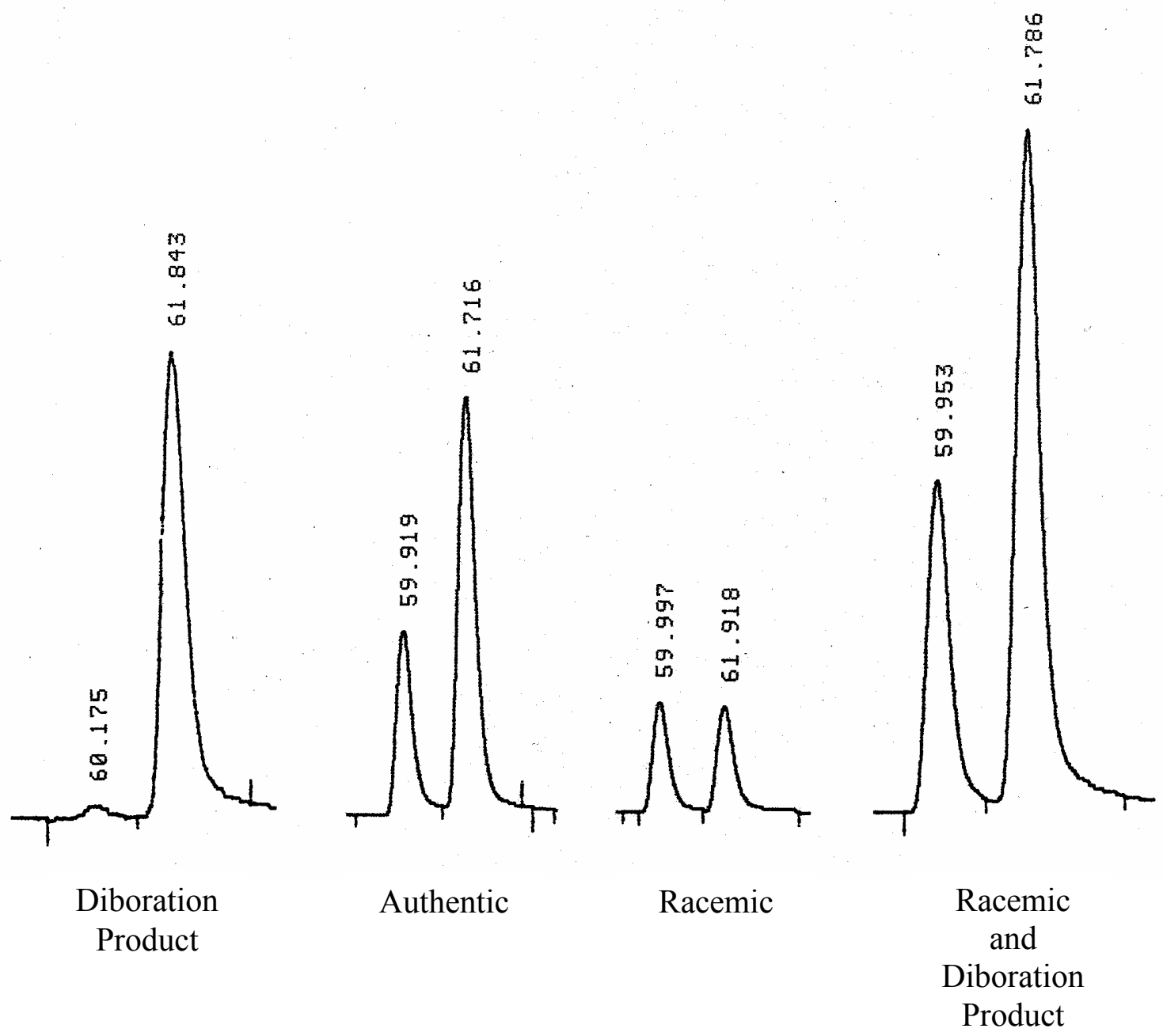




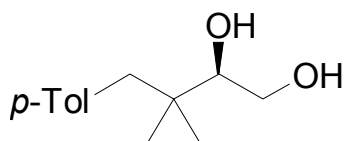

(R)-3,3-Dimethyl-4-p-tolyl-butane-1,2-diol. IR (neat): 3477 (br, s), 2987 (s), 3054 (s), 1277 (s) $\mathrm{cm}^{-1} ;{ }^{1} \mathrm{H}$ NMR: $\delta 7.07$ (2H, $\mathrm{d}, \mathrm{J}=8.6 \mathrm{~Hz}$, aromatic), $7.04(2 \mathrm{H}, \mathrm{d}, \mathrm{J}=8.6 \mathrm{~Hz}$, aromatic), 3.75 $\left(1 \mathrm{H}\right.$, ddd, J $\left.=10.7 \mathrm{~Hz}, 6.8 \mathrm{~Hz}, 3.0 \mathrm{~Hz}, \mathrm{CH}_{\mathrm{a}} \mathrm{H}_{\mathrm{b}} \mathrm{OH}\right), 3.55(1 \mathrm{H}$, ddd, J = 10.7 Hz, 9.4 Hz, 4.5 Hz, $\left.\mathrm{CH}_{\mathrm{a}} \mathbf{H}_{\mathbf{b}} \mathrm{OH}\right), 3.42(1 \mathrm{H}$, ddd, J = 9.4 Hz, 4.0 Hz, 3.0 Hz, $\mathrm{CHOH}), 2.71\left(1 \mathrm{H}, \mathrm{d}, \mathrm{J}=13.1 \mathrm{~Hz}\right.$, benzylic- $\left.\mathrm{CH}_{\mathrm{a}} \mathrm{H}_{\mathrm{b}}\right), 2.44(1 \mathrm{H}, \mathrm{d}, \mathrm{J}=13.1 \mathrm{~Hz}$, benzylic$\left.\mathrm{CH}_{\mathrm{a}} \mathbf{H}_{\mathrm{b}}\right), 2.30\left(3 \mathrm{H}, \mathrm{s}, \mathrm{PhCH}_{3}\right), 2.14(1 \mathrm{H}, \mathrm{d}, \mathrm{J}=4.0 \mathrm{~Hz}, \mathrm{CHOH}), 1.72(1 \mathrm{H}, \mathrm{dd}, \mathrm{J}=6.8 \mathrm{~Hz}$, $\left.4.5 \mathrm{~Hz}, \mathrm{CH}_{2} \mathrm{OH}\right), 0.89\left(3 \mathrm{H}, \mathrm{s}, \mathrm{CCH}_{3}\right), 0.82\left(3 \mathrm{H}, \mathrm{s}, \mathrm{CCH}_{3}\right) ;{ }^{13} \mathrm{C} \mathrm{NMR}: \delta 135.5,135.2$, 130.6, 128.6, 77.7, 63.1, 44.8, 37.3, 23.3, 22.5, 21.0. HRMS (FAB) Calc'd for $\mathrm{C}_{13} \mathrm{H}_{20} \mathrm{O}_{2}$ $(\mathrm{M}+\mathrm{Na})^{+}: 231.1356$. Found $(\mathrm{M}+\mathrm{Na})^{+}: 231.1353$.

Proof of Stereochemistry. Stereochemical ratios were determined by comparing to authentic racemic materials prepared by osmium tetraoxide catalyzed dihydroxylation. Absolute stereochemistry was established in comparison to authentic (R) isomer prepared via a Sharpless asymmetric dihydroxylation. (Becker, H.; King, S. B.; Taniguchi, M.; Vanhessche, K.; Sharpless, K. B. J. Org. Chem. 1995, 60, 3040).

SFC $\left(O D-H, 150 \mathrm{psi}, 50^{\circ} \mathrm{C}\right.$, flow $\left.=3 \mathrm{~mL} / \mathrm{min}, 1 \% \mathrm{MeOH}\right)$ analysis of diol product:

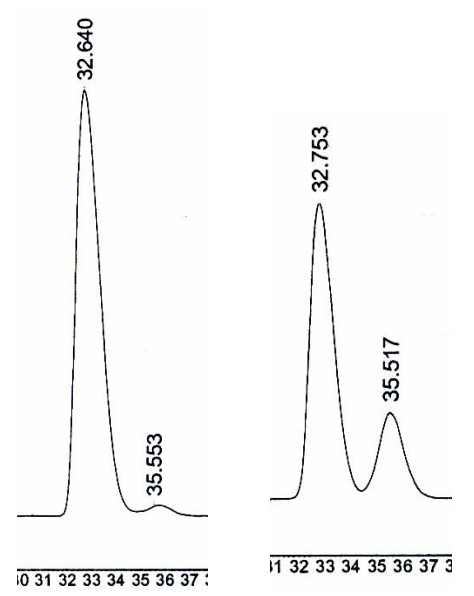

Diboration Authentic

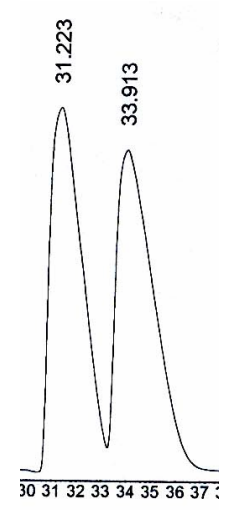

Racemic

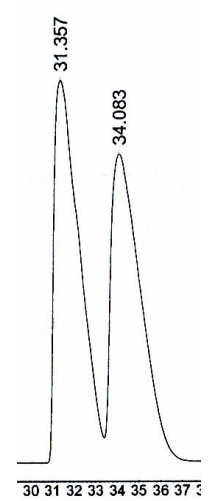

Racemic $+$

Diboration

Product 
(S)-3,3-Dimethyl-1-phenyl-butan-2-ol. IR (neat): 3489 (br, s), 1495 (w) $\mathrm{cm}^{-1} ;{ }^{1} \mathrm{H}$ NMR: $\delta$ 7.35-7.20 (5H, m, aromatic), $3.42(1 \mathrm{H}, \mathrm{dd}, \mathrm{J}=$ $10.4 \mathrm{~Hz}, 2.0 \mathrm{~Hz}, \mathrm{CHOH}), 2.90(1 \mathrm{H}, \mathrm{dd}, \mathrm{J}=13.6 \mathrm{~Hz}, 2.0 \mathrm{~Hz}$, $\left.\mathrm{CH}_{\mathbf{a}} \mathrm{H}_{\mathrm{b}} \mathrm{Ph}\right), 2.46\left(1 \mathrm{H}, \mathrm{dd}, \mathrm{J}=13.6 \mathrm{~Hz}, 10.4 \mathrm{~Hz}, \mathrm{CH}_{\mathrm{a}} \mathbf{H}_{\mathbf{b}} \mathrm{Ph}\right), 1.44(1 \mathrm{H}, \mathrm{br}$ s, OH) $0.99\left(9 \mathrm{H}, \mathrm{s}, \mathrm{C}\left(\mathrm{CH}_{3}\right)_{3}\right)$; ${ }^{13} \mathrm{C}$ NMR: $\delta 139.9,129.3,128.6,126.3,80.6,38.4,34.8$, 25.9. HRMS (FAB) Calc'd for $\left.\mathrm{C}_{12} \mathrm{H}_{18} \mathrm{O} \mathrm{M}+\mathrm{Na}\right)^{+}:$201.1250. Found $(\mathrm{M}+\mathrm{Na})^{+}$: 201.1250 .

Proof of Stereochemistry. Stereochemical ratios were determined in comparison to authentic enantioenriched material prepared via Dess-Martin periodinane oxidation followed by sodium borohydride reduction of enantiopure carbohydroxylation product. Absolute stereochemistry established by analogy to the corresponding diol product.

Chiral GLC ( $\beta$-dex, Supelco, $\left.100^{\circ} \mathrm{C}\right)$ analysis of alcohol product:

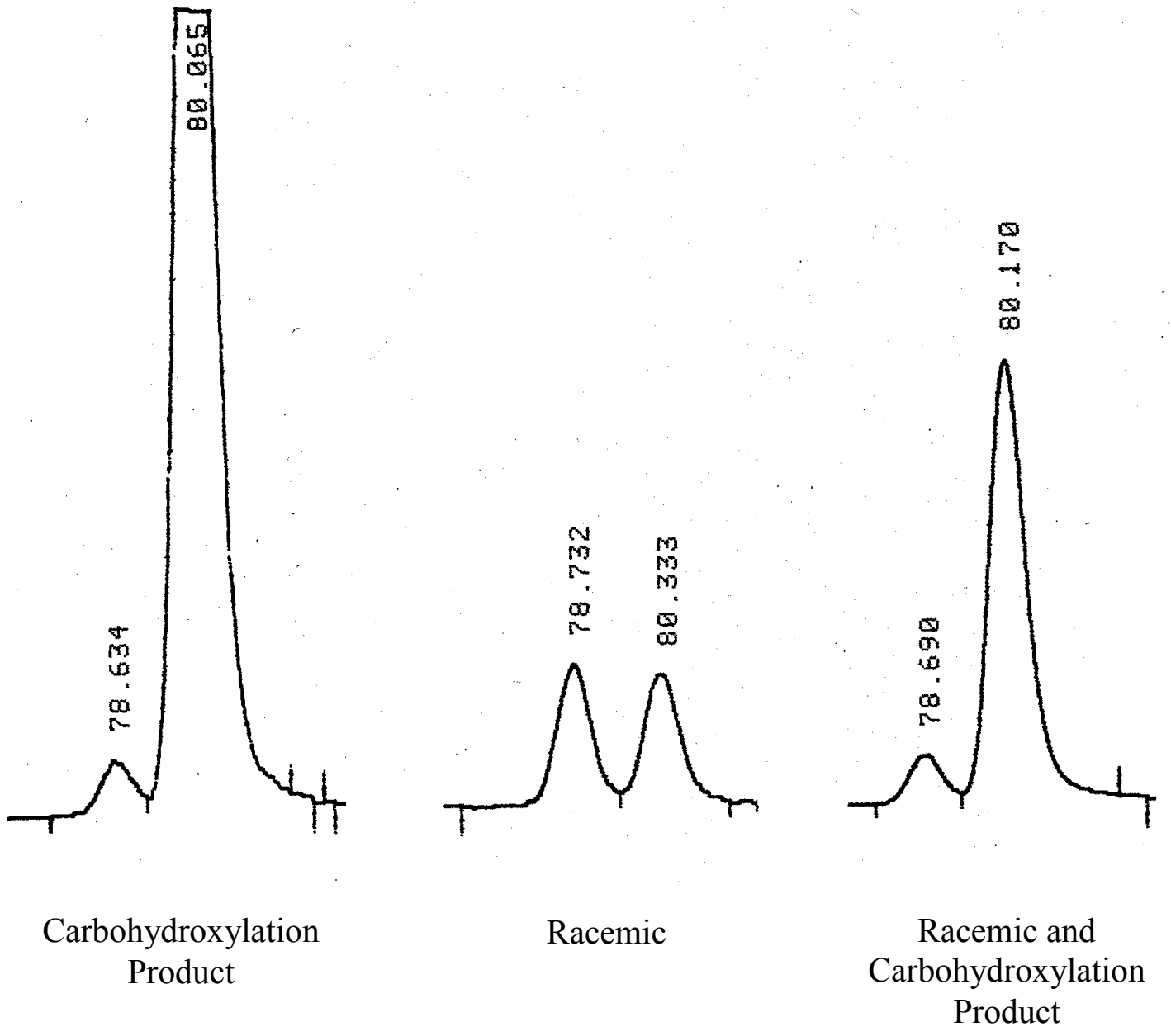


(S)-4-Benzyloxy-3,3-dimethyl-1-pyridin-3-yl-butan-2-

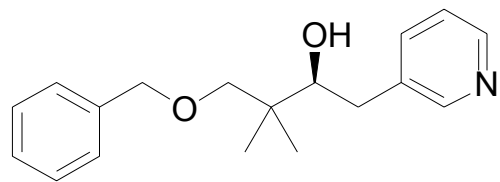

ol. IR (neat): 3479 (br, s), $1270(\mathrm{~m}) \mathrm{cm}^{-1} ;{ }^{1} \mathrm{H}$ NMR: $\delta 8.44$ $(1 \mathrm{H}, \mathrm{d}, \mathrm{J}=1.6 \mathrm{~Hz}$, pyridyl), $8.41(1 \mathrm{H}, \mathrm{dd}, \mathrm{J}=4.8 \mathrm{~Hz}, 1.6$ $\mathrm{Hz}$, pyridyl), $7.58(1 \mathrm{H}, \mathrm{dt}, \mathrm{J}=7.6 \mathrm{~Hz}, 1.6 \mathrm{~Hz}$, pyridyl), 7.35-7.25 (5H, m, aromatic), $7.19(1 \mathrm{H}, \mathrm{dd}, \mathrm{J}=7.6 \mathrm{~Hz}, 4.8$

$\mathrm{Hz}$, pyridyl), $4.50\left(1 \mathrm{H}, \mathrm{d}, \mathrm{J}=19.2 \mathrm{~Hz}, \mathrm{CH}_{\mathrm{a}} \mathrm{H}_{\mathrm{b}} \mathrm{Ph}\right), 4.47\left(1 \mathrm{H}, \mathrm{d}, \mathrm{J}=19.2 \mathrm{~Hz}, \mathrm{CH}_{\mathrm{a}} \mathbf{H}_{\mathbf{b}} \mathrm{Ph}\right)$, $3.65(1 \mathrm{H}, \mathrm{dd}, \mathrm{J}=10.4 \mathrm{~Hz}, 2.0 \mathrm{~Hz}, \mathrm{CHOH}), 3.42\left(1 \mathrm{H}, \mathrm{d}, \mathrm{J}=4.8 \mathrm{~Hz}, \mathrm{OCH}_{\mathbf{a}} \mathrm{H}_{\mathrm{b}} \mathrm{C}\right), 3.33$ $\left(1 \mathrm{H}, \mathrm{d}, \mathrm{J}=4.8 \mathrm{~Hz}, \mathrm{OCH}_{\mathrm{a}} \mathbf{H}_{\mathrm{b}} \mathrm{C}\right), 2.77\left(1 \mathrm{H}, \mathrm{dd}, \mathrm{J}=13.8 \mathrm{~Hz}, 2.0 \mathrm{~Hz}, \mathrm{CH}_{\mathrm{a}} \mathrm{H}_{\mathrm{b}}(\mathrm{Pyr})\right), 2.50$ $\left(1 \mathrm{H}, \mathrm{dd}, \mathrm{J}=13.8 \mathrm{~Hz}, 10.4 \mathrm{~Hz}, \mathrm{CH}_{\mathrm{a}} \mathbf{H}_{\mathrm{b}}(\mathrm{Pyr})\right), 1.01\left(3 \mathrm{H}, \mathrm{s}, \mathbf{C C H}_{3}\right), 0.99\left(3 \mathrm{H}, \mathrm{s}, \mathrm{CCH}_{3}\right) ;{ }^{13} \mathrm{C}$ NMR: $\delta 150.5,147.3,137.7,137.0,135.8,128.4,127.8,127.6,123.1,79.5,79.0,73.6$, 38.5, 35.4, 22.8, 19.7. HRMS (FAB) Calc'd for $\mathrm{C}_{18} \mathrm{H}_{23} \mathrm{NO}_{2}(\mathrm{M}+\mathrm{Na})^{+}$: 308.1621 Found $(\mathrm{M}+\mathrm{Na})^{+}: 308.1614$.

Proof of Stereochemistry. Stereochemical ratios were determined in comparison to authentic enantioenriched material prepared via Dess-Martin periodinane oxidation followed by sodium borohydride reduction. Incomplete oxidation of the enantiopure carbohydroxylation product led to the enatioenriched material used for analysis. Absolute stereochemistry established by analogy to structurally similar diol product obtained by the diboration/oxidation procedure.

Chiral HPLC (Chiralcel-OD, Daicel, 5.0\% iPrOH in hexanes, $1.0 \mathrm{~mL} / \mathrm{min}$ ) analysis of alcohol product:

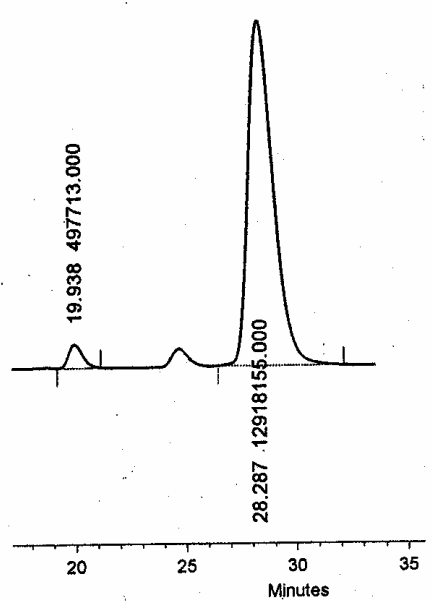

Carbohydroxylation

Product

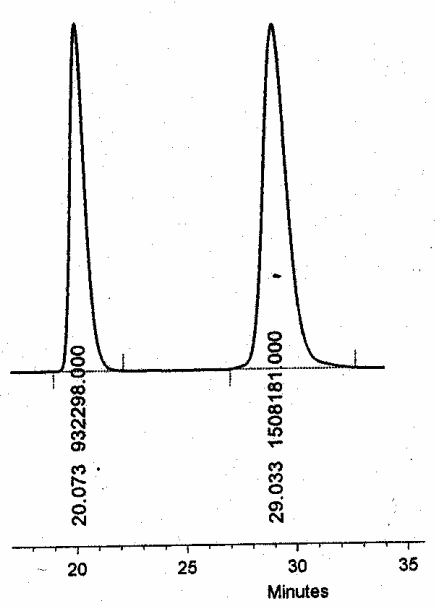

Enantioenriched

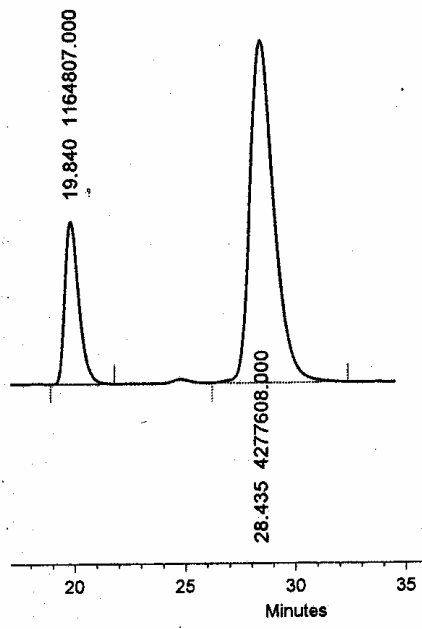

Enantioenriched and Carbohydroxylation Product 


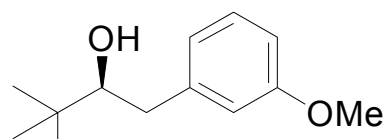

(S)-1-(3-Methoxy-phenyl)-3,3-dimethyl-butan-2-ol. IR (neat): 3464 (br, s), $1490(\mathrm{w}), 1272(\mathrm{~m}) \mathrm{cm}^{-1} ;{ }^{1} \mathrm{H}$ NMR: $\delta$ 7.25-7.18 (1H, $\mathrm{m}$, aromatic), 6.83-6.75 (3H, m, aromatic), $3.79\left(3 \mathrm{H}, \mathrm{s}, \mathrm{OCH}_{3}\right)$, $3.42(1 \mathrm{H}, \mathrm{d}, \mathrm{J}=10.4 \mathrm{~Hz}, \mathrm{CHOH}), 2.87(1 \mathrm{H}, \mathrm{d}, \mathrm{J}=13.6 \mathrm{~Hz}$, $\left.\mathrm{CH}_{\mathbf{a}} \mathrm{H}_{\mathrm{b}} \mathrm{Ar}\right), 2.43\left(1 \mathrm{H}, \mathrm{dd}, \mathrm{J}=13.6 \mathrm{~Hz}, 10.4 \mathrm{~Hz}, \mathrm{CH}_{\mathrm{a}} \mathbf{H}_{\mathbf{b}} \mathrm{Ar}\right), 1.50(1 \mathrm{H}, \mathrm{br} \mathrm{s}, \mathrm{OH}) 0.99(9 \mathrm{H}$, s, $\left.\mathrm{C}\left(\mathrm{CH}_{3}\right)_{3}\right) ;{ }^{13} \mathrm{C}$ NMR: $\delta 159.8,141.5,129.5,121.6,114.9,111.7,80.5,55.1,38.4,34.8$, 25.8. HRMS (FAB) Calc'd for $\mathrm{C}_{13} \mathrm{H}_{20} \mathrm{O}_{2}(\mathrm{M}+\mathrm{Na})^{+}: 231.1356$ Found $(\mathrm{M}+\mathrm{Na})^{+}$: 231.1361 .

Proof of Stereochemistry. Stereochemical ratios were determined in comparison to alcohol obtained by mixing carbohydroxylation products produced by opposite enantiomers of Quinap. Absolute stereochemistry established by analogy to the corresponding diol product.

Chiral GLC ( $\beta$-dex, Supelco, $\left.130^{\circ} \mathrm{C}\right)$ analysis of alcohol product:

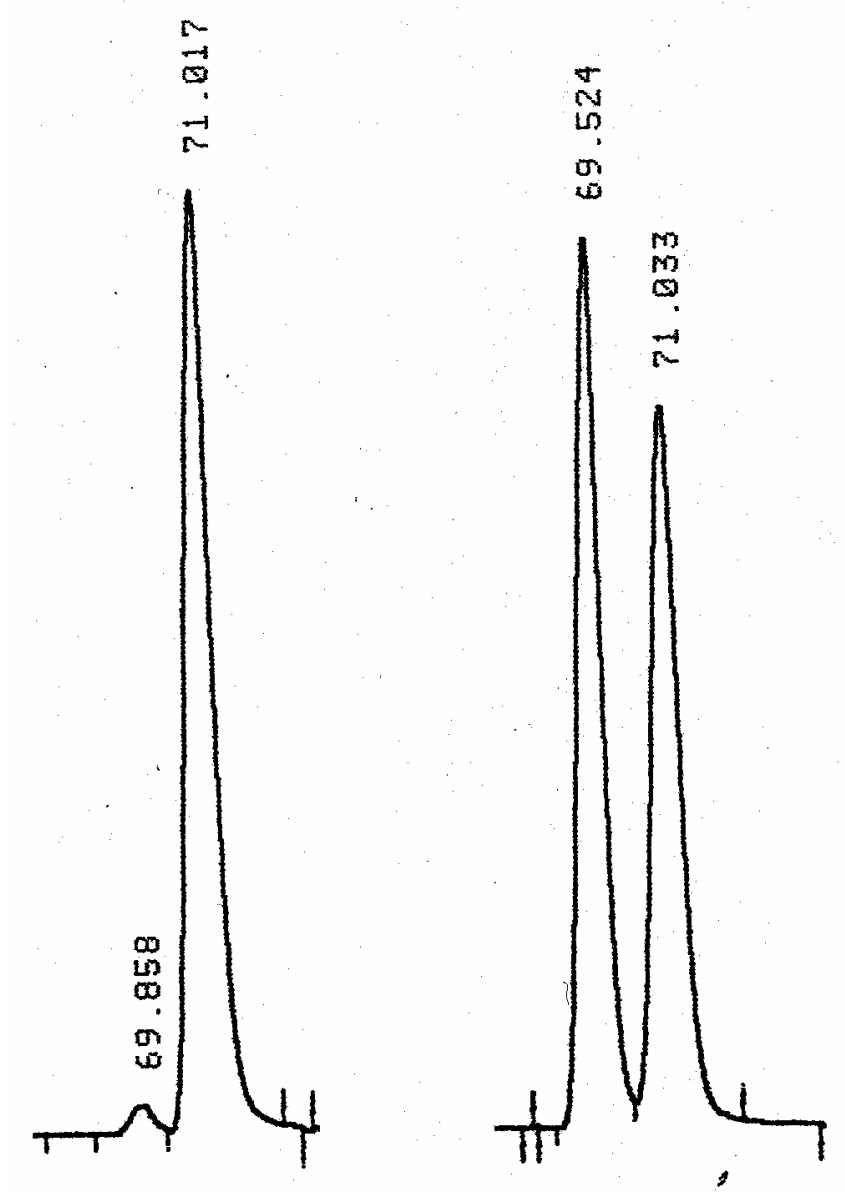

Carbohydroxylation

Enantiomer mixture

Product 
(S)-3,3-Dimethyl-1-napthalen-2-yl-l-butan-2-ol (Obtained with $R$-Quinap as ligand). IR (neat): 3465 (br, s), 3050 (s), 1273 (s) $\mathrm{cm}^{-1} ;{ }^{1} \mathrm{H}$ NMR: $\delta$ 7.76-7.82 (3H, m, aromatic), $7.68(1 \mathrm{H}, \mathrm{s}$, aromatic), 7.40-7.47 $(2 \mathrm{H}, \mathrm{m}$, aromatic), $7.36(1 \mathrm{H}, \mathrm{dd}, \mathrm{J}=9.0 \mathrm{~Hz}$, $1.7 \mathrm{~Hz}$, aromatic), $3.53(1 \mathrm{H}$, ddd, $\mathrm{J}=10.8 \mathrm{~Hz}, 3.4 \mathrm{~Hz}, 2.0 \mathrm{~Hz}$, $\mathrm{CHOH}), 3.07\left(1 \mathrm{H}, \mathrm{dd}, \mathrm{J}=13.6 \mathrm{~Hz}, 2.0 \mathrm{~Hz}, \mathrm{CH}_{\mathrm{a}} \mathrm{H}_{\mathrm{b}}\right), 2.63(1 \mathrm{H}, \mathrm{dd}, \mathrm{J}=13.6 \mathrm{~Hz}, 10.8 \mathrm{~Hz}$, $\left.\mathrm{CH}_{\mathrm{a}} \mathbf{H}_{\mathrm{b}}\right), 1.46(1 \mathrm{H}, \mathrm{d}, 3.4 \mathrm{~Hz}, \mathrm{COH}), 1.03\left(9 \mathrm{H}, \mathrm{s}, \mathrm{C}\left(\mathrm{CH}_{3}\right)_{3}\right) ;{ }^{13} \mathrm{C}$ NMR: $\delta$ 137.8, 134.0, 132.6, 128.7, 128.2, 128.1, 128.0, 127.9, 126.5, 125.8, 80.8, 39.0, 35.3, 26.3. HRMS (FAB) Calc'd for $\mathrm{C}_{16} \mathrm{H}_{20} \mathrm{O}(\mathrm{M}+\mathrm{Na})^{+}: 251.1407$. Found $(\mathrm{M}+\mathrm{Na})^{+}: 251.1408$.

Proof of Stereochemistry. Stereochemical ratios were determined in comparison to authentic mixture of enantiomers prepared via Dess-Martin oxidation followed by sodium borohydride reduction of enantioenriched carbohydroxylation product. Absolute stereochemistry established by analogy to the corresponding diol product.

SFC (OD-H, $150 \mathrm{psi}, 50{ }^{\circ} \mathrm{C}$, flow $\left.=3 \mathrm{~mL} / \mathrm{min}, 1 \% \mathrm{MeOH}\right)$ analysis of alcohol product:

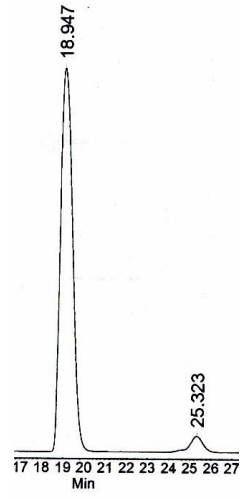

Carbohydroxylation

Product

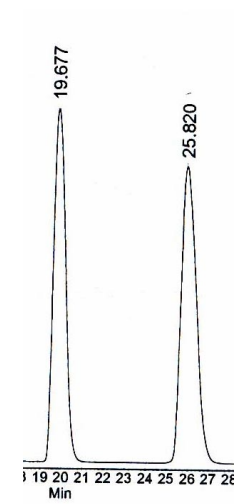

Racemic

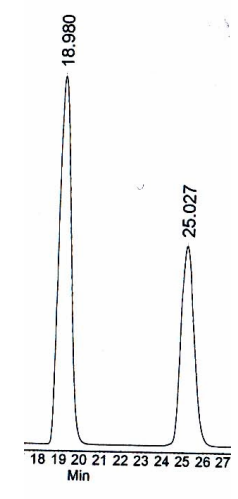

Racemic $+$

Carbohydroxylation

Product 


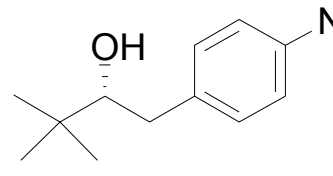

$\mathrm{NO}_{2}$ (S)-3,3-Dimethyl-1-(4-nitr9so-phenyl)-butan-2-ol

(Obtained with $R$-Quinap as ligand). IR (neat): 3458 (br, s), 3056 (s), 2989 (s), 1520 (s), 1349 (s), $1272(\mathrm{~s}) \mathrm{cm}^{-1} ;{ }^{1} \mathrm{H}$ NMR: $\delta 8.15(2 \mathrm{H}, \mathrm{d}, \mathrm{J}$ $=8.7 \mathrm{~Hz}$, aromatic $), 7.40(2 \mathrm{H}, \mathrm{d}, \mathrm{J}=8.7 \mathrm{~Hz}$, aromatic $), 3.44(1 \mathrm{H}$, ddd, J = 10.6 Hz, 4.5 Hz, 2.0 Hz, CHOH), $2.95\left(1 \mathrm{H}, \mathrm{dd}, \mathrm{J}=13.7 \mathrm{~Hz}, 1.3 \mathrm{~Hz}, \mathrm{PhCH}_{\mathrm{a}} \mathrm{H}_{\mathrm{b}}\right.$ ), $2.61\left(1 \mathrm{H}, \mathrm{dd}, \mathrm{J}=13.7 \mathrm{~Hz}, 10.6 \mathrm{~Hz}, \mathrm{PhCH}_{\mathrm{a}} \mathbf{H}_{\mathbf{b}}\right), 1.40(1 \mathrm{H}, \mathrm{d}, \mathrm{J}=4.5 \mathrm{~Hz}, \mathrm{COH}), 0.99(9 \mathrm{H}$, $\mathrm{s}, \mathrm{C}\left(\mathrm{CH}_{3}\right)_{3} ;{ }^{13} \mathrm{C}$ NMR: $\delta 148.8,146.9,130.5,123.9,80.8,38.5,35.5,26.0$. HRMS (FAB) Calc'd for $\mathrm{C}_{12} \mathrm{H}_{17} \mathrm{O}_{3}(\mathrm{M}+\mathrm{Na})^{+}$: 246.1101. Found $(\mathrm{M}+\mathrm{Na})^{+}$: 246.1106 .

Proof of Stereochemistry. Stereochemical ratios were determined by SFC analysis in comparison to enantiomer mixtures. Enantiomer mixtures were obtained by mixing carbohydroxylation products obtained from reactions using opposite enantiomers of Quinap. Absolute stereochemistry established by analogy to the corresponding diol product.

SFC (OD-H, $150 \mathrm{psi}, 50{ }^{\circ} \mathrm{C}$, flow $\left.=3 \mathrm{~mL} / \mathrm{min}, 0 \% \mathrm{MeOH}\right)$ analysis of alcohol product:

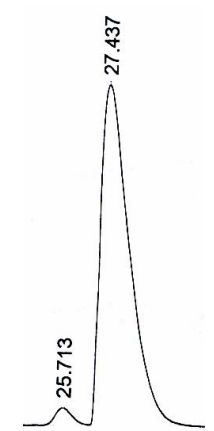

$\longdiv { 2 5 2 6 2 7 2 8 2 9 3 0 3 1 3 }$

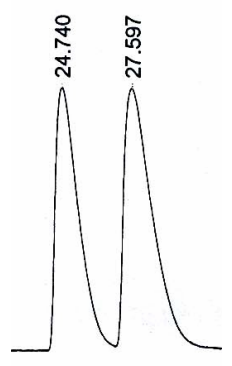

232425262728293031

Carbohydroxylation Enantiomer mixture

Products 


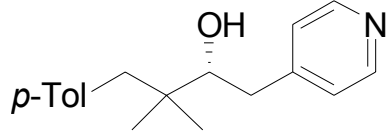

(S)-3,3-Dimethyl-1-pyridin-4-yl-4-p-tolyl-butan-2-ol (Obtained with $R$-Quinap as ligand). IR (neat): 3477 (br, s), 3056 (s) $2989(\mathrm{~s}), 1272(\mathrm{~s}) \mathrm{cm}^{-1} ;{ }^{1} \mathrm{H}$ NMR: $\delta 8.48(2 \mathrm{H}, \mathrm{d}, \mathrm{J}=5.9 \mathrm{~Hz}$, pyridyl), $7.14(2 \mathrm{H}, \mathrm{d}, \mathrm{J}=5.9 \mathrm{~Hz}$, pyridyl), $7.08(2 \mathrm{H}, \mathrm{d}, \mathrm{J}=8.5 \mathrm{~Hz}$, aromatic), $7.05(2 \mathrm{H}$, d, J $=8.5 \mathrm{~Hz}$, aromatic), $3.49(1 \mathrm{H}, \mathrm{br} \mathrm{d}, \mathrm{J}=10.6 \mathrm{~Hz}, \mathrm{CHOH}), 2.89(1 \mathrm{H}, \mathrm{dd}, \mathrm{J}=13.6 \mathrm{~Hz}$, $\left.1.7 \mathrm{~Hz}, \mathrm{CHOHCH}_{\mathrm{a}} \mathrm{H}_{\mathrm{b}}\right), 2.75\left(1 \mathrm{H}, \mathrm{d}, \mathrm{J}=13.1 \mathrm{~Hz}\right.$, benzylic-C $\left.\mathbf{C H}_{\mathrm{a}} \mathrm{H}_{\mathrm{b}}\right), 2.56(1 \mathrm{H}, \mathrm{dd} \mathrm{J}=13.6$ $\left.\mathrm{Hz}, 10.6 \mathrm{~Hz}, \mathrm{CHOHCH}_{\mathrm{a}} \mathbf{H}_{\mathbf{b}}\right), 2.54\left(1 \mathrm{H}, \mathrm{d}, \mathrm{J}=13.1 \mathrm{~Hz}\right.$, benzylic- $\left.\mathrm{CH}_{\mathrm{a}} \mathbf{H}_{\mathbf{b}}\right) 2.31(3 \mathrm{H}, \mathrm{s}$, $\left.\mathrm{PhCH}_{3}\right), 0.97\left(3 \mathrm{H}, \mathrm{s}, \mathrm{CCH}_{3}\right), 0.92\left(3 \mathrm{H}, \mathrm{s}, \mathrm{CCH}_{3}\right) ;{ }^{13} \mathrm{C} \mathrm{NMR}: \delta 149.6,149.5,135.5$, 135.4, 130.6, 128.5, 124.9, 78.0, 44.4, 38.9, 37.7, 23.6, 22.4, 21.0. HRMS (FAB) Calc'd for $\mathrm{C}_{18} \mathrm{H}_{23} \mathrm{NO}(\mathrm{M}+\mathrm{Na})^{+}: 270.1852$. Found $(\mathrm{M}+\mathrm{Na})^{+}: 270.1856$.

Proof of Stereochemistry. Stereochemical ratios were determined by SFC analysis in comparison to enantiomer mixtures. Enantiomer mixtures were obtained by mixing carbohydroxylation products obtained from reactions using opposite enantiomers of Quinap. Absolute stereochemistry established by analogy to the corresponding diol product.

SFC $\left(O D-H, 150 \mathrm{psi}, 50^{\circ} \mathrm{C}\right.$, flow $\left.=3.5 \mathrm{~mL} / \mathrm{min}, 3 \% \mathrm{MeOH}\right)$ analysis of alcohol product:

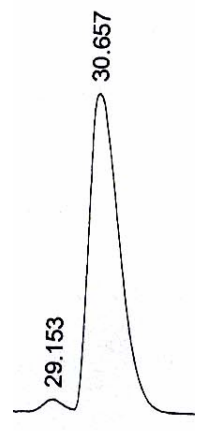

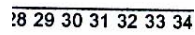

Carbohydroxylation

Products

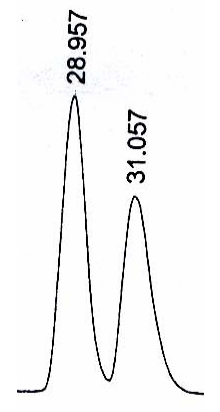

282930313233

Enantiomer mixture 


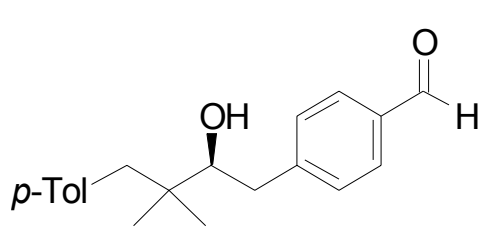

(R)-4-(2-Hydroxy-3,3-dimethyl-4-p-tolyl-butyl)-benzaldhyde. IR (neat): 3487 (br, s), 3056 (s), 2989 (s), 1696 (s), H $1272(\mathrm{~s}) \mathrm{cm}^{-1} ;{ }^{1} \mathrm{H}$ NMR: $\delta 9.97(1 \mathrm{H}, \mathrm{s}, \mathrm{PhCOH}), 7.81(2 \mathrm{H}$, $\mathrm{d}, \mathrm{J}=8.1 \mathrm{~Hz}$, formylphenyl), $7.38(2 \mathrm{H}, \mathrm{d}, \mathrm{J}=8.1 \mathrm{~Hz}$, formylphenyl), $7.08(2 \mathrm{H}, \mathrm{d}, \mathrm{J}=8.9 \mathrm{~Hz}$, aromatic $), 7.05$ $(2 \mathrm{H}, \mathrm{d}, \mathrm{J}=8.9 \mathrm{~Hz}$, aromatic), $3.51(1 \mathrm{H}, \mathrm{m}, \mathrm{CHCOH}), 2.99(1 \mathrm{H}, \mathrm{dd}, \mathrm{J}=13.5 \mathrm{~Hz}, 1.5 \mathrm{~Hz}$, $\left.\mathrm{CHOHCH}_{\mathrm{a}} \mathrm{H}_{\mathrm{b}}\right), 2.75\left(1 \mathrm{H}, \mathrm{d}, \mathrm{J}=13.1 \mathrm{~Hz}\right.$, benzylic- $\left.\mathrm{CH}_{\mathrm{a}} \mathrm{H}_{\mathrm{b}}\right), 2.65(1 \mathrm{H}, \mathrm{dd}, \mathrm{J}=13.5 \mathrm{~Hz}$, $\left.10.8 \mathrm{~Hz}, \mathrm{CHOHCH}_{\mathrm{a}} \mathbf{H}_{\mathbf{b}}\right), 2.55\left(1 \mathrm{H}, \mathrm{d}, \mathrm{J}=13.1 \mathrm{~Hz}\right.$, benzylic- $\left.\mathrm{CH}_{\mathbf{a}} \mathbf{H}_{\mathbf{b}}\right), 1.40(1 \mathrm{H}, \mathrm{d}, \mathrm{J}=4.3$ $\left.\mathrm{Hz}, \mathrm{CCCH}_{2} \mathrm{OH}\right), 0.98\left(3 \mathrm{H}, \mathrm{s}, \mathrm{CCH}_{3}\right), 0.93\left(3 \mathrm{H}, \mathrm{s}, \mathrm{CCH}_{3}\right) ;{ }^{13} \mathrm{C}$ NMR: $\delta 191.9,147.6$, 135.5, 135.3, 134.8, 130.6, 130.1, 130.0, 128.6, 78.6, 44.4, 38.8, 38.4, 23.5, 22.4, 21.0. HRMS (FAB) Calc'd for $\mathrm{C}_{20} \mathrm{H}_{24} \mathrm{O}_{2}(\mathrm{M}+\mathrm{Na})^{+}: 319.1669$. Found $(\mathrm{M}+\mathrm{Na})^{+}: 319.1667$.

Proof of Stereochemistry. Stereochemical ratios were determined by SFC analysis in comparison to enantiomer mixtures. Enantiomer mixtures were obtained by mixing carbohydroxylation products obtained from reactions using opposite enantiomers of Quinap. Absolute stereochemistry established by analogy to the corresponding diol product.

SFC $\left(O D-H, 150 \mathrm{psi}, 50{ }^{\circ} \mathrm{C}\right.$, flow $\left.=4 \mathrm{~mL} / \mathrm{min}, 2 \% \mathrm{MeOH}\right)$ analysis of alcohol product:
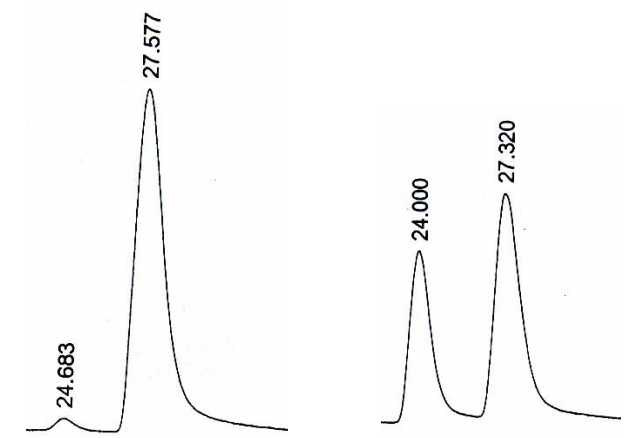

24252627282930313233

$2 3 \longdiv { 2 4 2 5 2 6 2 7 2 8 2 9 3 0 3 1 3 }$

Carbohydroxylation

Enantiomer mixture

Products 


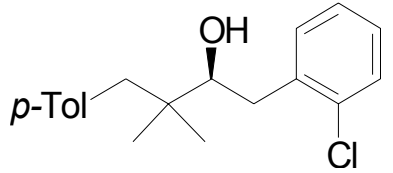

(R)-1-(2-Chloro-phenyl)-3,3-dimethyl-4-p-tolyl-butan-2-ol. IR (neat): 3487 (br, s), 3056 (s), 2989 (s), 1272 (s) $\mathrm{cm}^{-1} ;{ }^{1} \mathrm{H}$ NMR: $\delta 7.36(1 \mathrm{H}, \mathrm{d}, \mathrm{J}=7.5 \mathrm{~Hz}$, aromatic), $7.28(1 \mathrm{H}, \mathrm{d}, \mathrm{J}=6.9 \mathrm{~Hz}$, aromatic), $7.24(1 \mathrm{H}, \mathrm{d}$, aromatic), 7.14-7.25 (2H, $\mathrm{m}$, aromatic), $3.59(1 \mathrm{H}, \mathrm{m}, \mathrm{CHOH}), 3.17\left(1 \mathrm{H}, \mathrm{d}, \mathrm{J}=13.6 \mathrm{~Hz}, \mathrm{Cl}-\mathrm{PhCH}_{\mathrm{a}} \mathrm{H}_{\mathrm{b}}\right), 2.76(1 \mathrm{H}, \mathrm{d}, \mathrm{J}=13.0 \mathrm{~Hz}$, benzylic- $\left.\mathrm{CH}_{\mathbf{a}} \mathrm{H}_{\mathrm{b}}\right), 2.67\left(1 \mathrm{H}, \mathrm{m}, \mathrm{J}=13.6 \mathrm{~Hz}, \mathrm{Cl}_{-} \mathrm{PhCH}_{\mathrm{a}} \mathbf{H}_{\mathbf{b}}\right), 2.59(1 \mathrm{H}, \mathrm{d}, \mathrm{J}=13.0 \mathrm{~Hz}$, benzylic- $\left.\mathrm{CH}_{\mathrm{a}} \mathbf{H}_{\mathbf{b}}\right), 2.32\left(3 \mathrm{H}, \mathrm{s}, \mathrm{PhCH}_{3}\right), 1.47-1.49(1 \mathrm{H}, \mathrm{m}, \mathrm{CHOH}) 0.99\left(3 \mathrm{H}, \mathrm{s}, \mathrm{CCH}_{3}\right)$, $0.94\left(3 \mathrm{H}, \mathrm{s}, \mathrm{CCH}_{3}\right) ;{ }^{13} \mathrm{C}$ NMR: $\delta 137.5,135.6,135.3,134.3,131.9,130.7,129.6,128.5$, 127.8, 126.7, 77.5, 44.0, 39.0, 35.9, 23.3, 22.1, 21.0. HRMS (FAB) Calc'd for $\mathrm{C}_{19} \mathrm{H}_{23} \mathrm{ClO}(\mathrm{M}+\mathrm{Na})^{+}: 325.1330$. Found $(\mathrm{M}+\mathrm{Na})^{+}: 325.1325$.

(R)-2-(1,1-Dimethyl-2-p-tolyl-ethyl)-2,3-dihydrobenzofuran. IR (neat): 3494 (br, s), 3056 (s), 2989 (s) $\mathrm{cm}^{-1} ;{ }^{1} \mathrm{H}$ NMR: $\delta$ 7.05-7.15 (6H, m, aromatic), 6.77-6.82 $(2 \mathrm{H}, \mathrm{m}$, aromatic), $4.48\left(1 \mathrm{H}, \mathrm{t}, \mathrm{CHCCH}_{2} \mathrm{O}\right), 3.03(1 \mathrm{H}, \mathrm{dd}, \mathrm{J}=15.8 \mathrm{~Hz}$, p-Tol

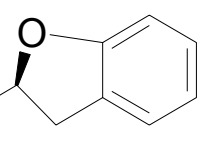
$\left.9.1 \mathrm{~Hz}, \mathrm{CHOCH}_{\mathbf{a}} \mathrm{H}_{\mathrm{b}}\right), 3.09\left(1 \mathrm{H}, \mathrm{dd}, \mathrm{J}=15.8 \mathrm{~Hz}, 9.5 \mathrm{~Hz}, \mathrm{CHOCH}_{\mathrm{a}} \mathbf{H}_{\mathbf{b}}\right), 2.74(1 \mathrm{H}, \mathrm{d}, \mathbf{J}=$
$13.1 \mathrm{~Hz}$, benzylic- $\left.\mathbf{C H}_{\mathbf{a}} \mathrm{H}_{\mathrm{b}}\right), 2.56\left(1 \mathrm{H}, \mathrm{d}, \mathrm{J}=13.1 \mathrm{~Hz}\right.$, benzylic- $\left.\mathrm{CH}_{\mathrm{a}} \mathbf{H}_{\mathbf{b}}\right), 2.31(3 \mathrm{H}, \mathrm{s}$, $\left.\mathrm{PhCH}_{3}\right), 0.93\left(3 \mathrm{H}, \mathrm{s}, \mathrm{CCH}_{3}\right), 0.84\left(3 \mathrm{H}, \mathrm{s}, \mathrm{CCH}_{3}\right) ;{ }^{13} \mathrm{C} \mathrm{NMR}: \delta 160.1,135.5,135.0$, 130.7, 128.5, 127.8, 127.1, 124.7, 119.9, 109.0, 88.7, 44.3, 38.0, 30.6, 22.1, 21.8, 21.0. HRMS (FAB) Calc'd for $\mathrm{C}_{19} \mathrm{H}_{22} \mathrm{O}(\mathrm{M}+\mathrm{Na})^{+}: 289.1563$. Found $(\mathrm{M}+\mathrm{Na})^{+}: 289.1566$.

Proof of Stereochemistry. Stereochemical ratios were determined in comparison to furan obtained by mixing reaction products produced by opposite enantiomers of Quinap. Absolute stereochemistry established by analogy to the corresponding diol product.

SFC (AD-H, $150 \mathrm{psi}, 50^{\circ} \mathrm{C}$, flow $\left.=2 \mathrm{~mL} / \mathrm{min}, 1 \% \mathrm{MeOH}\right)$ analysis of ether product:

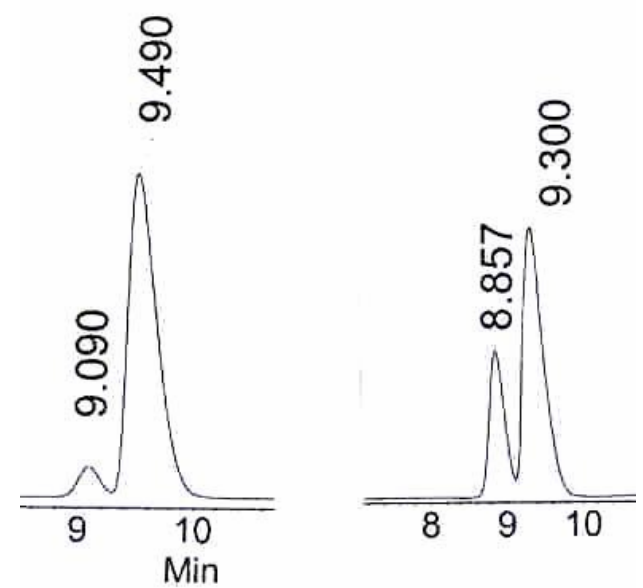

Reaction Product Enantiomer mixture 

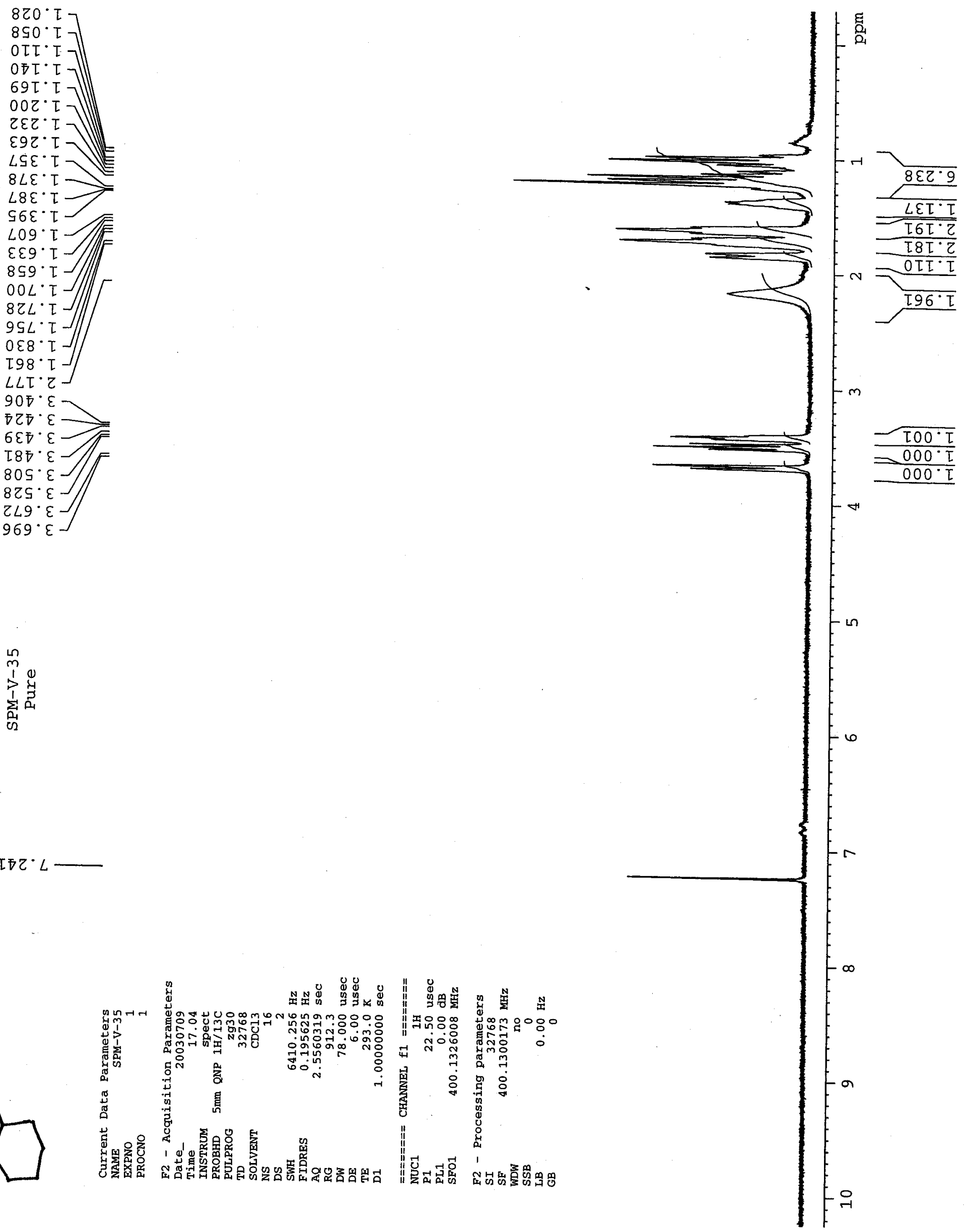

I万乙 $\cdot L$

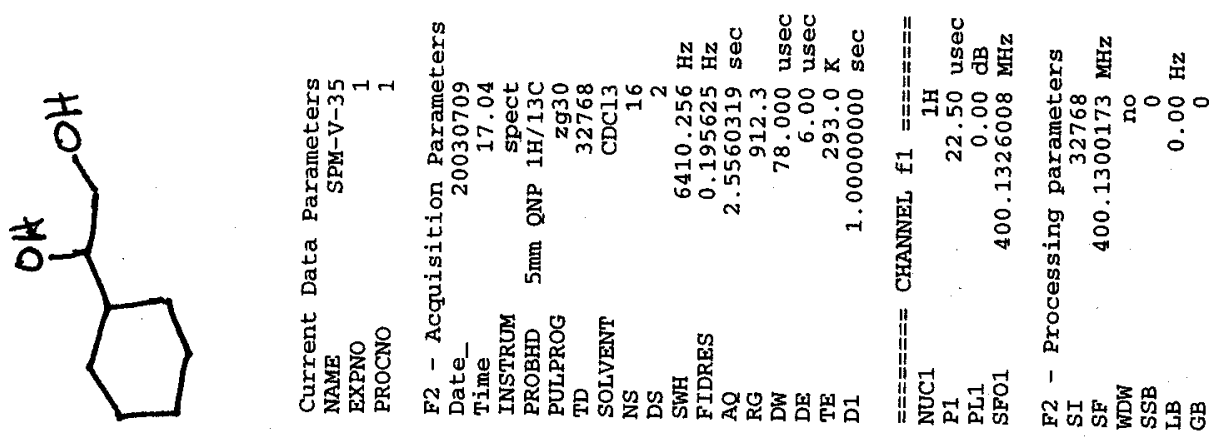



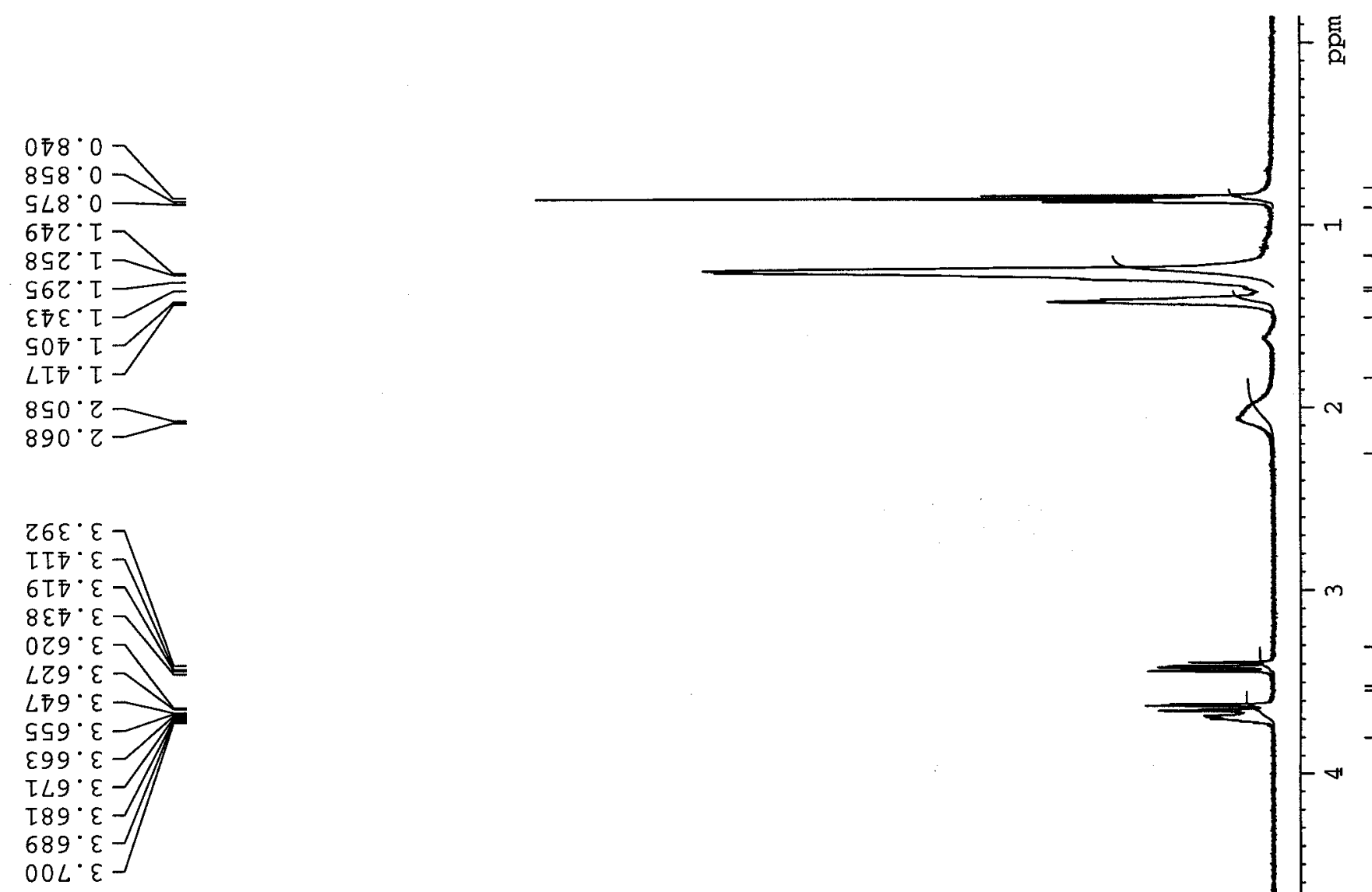

$=\quad z Z \cdot \varepsilon$

$\frac{\varepsilon 99^{\circ} \tau \tau}{876^{\circ} \tau}$

$890^{\circ} \mathrm{Z}$

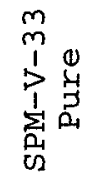

$\tau \nabla \tau^{\circ} L$
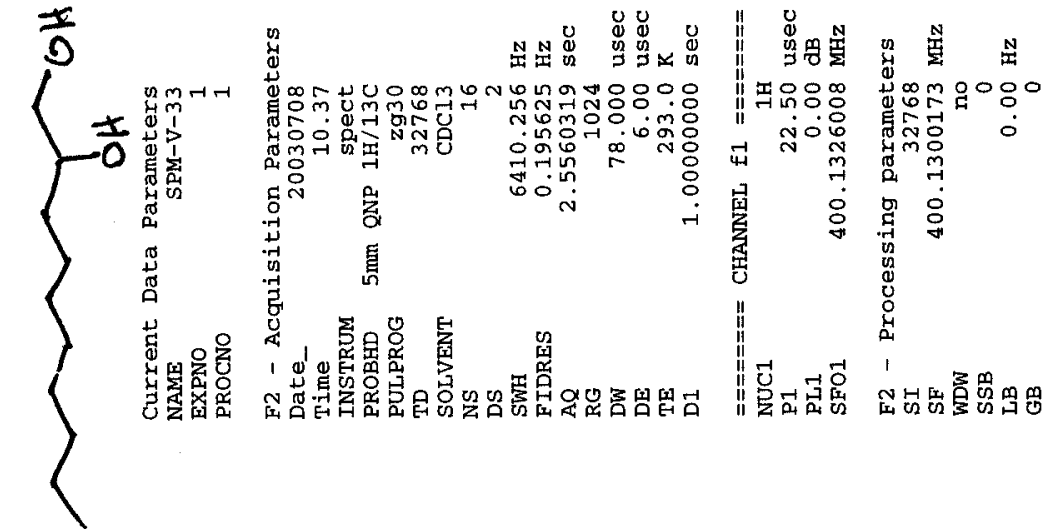

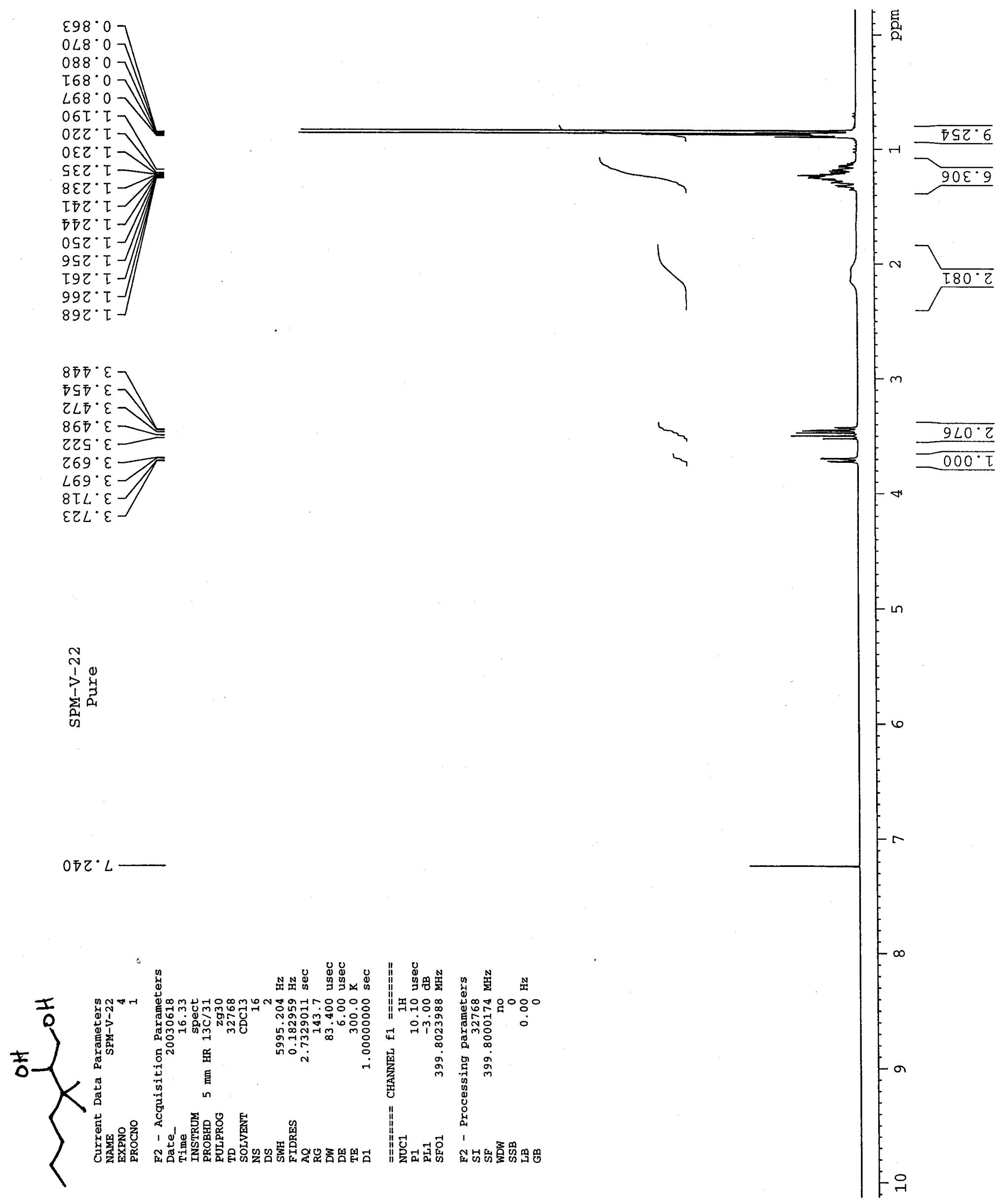

Page 20 

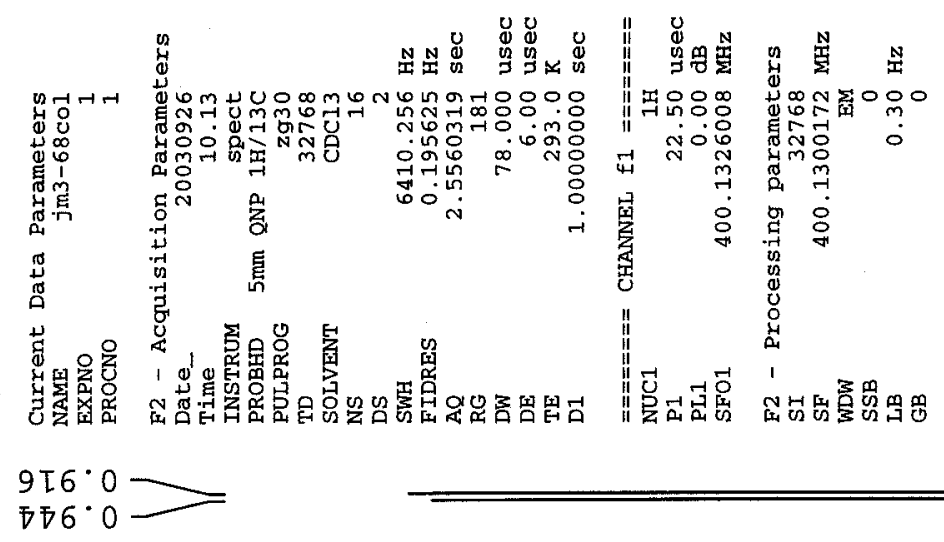

$s \varepsilon \tau^{\circ} \tau$
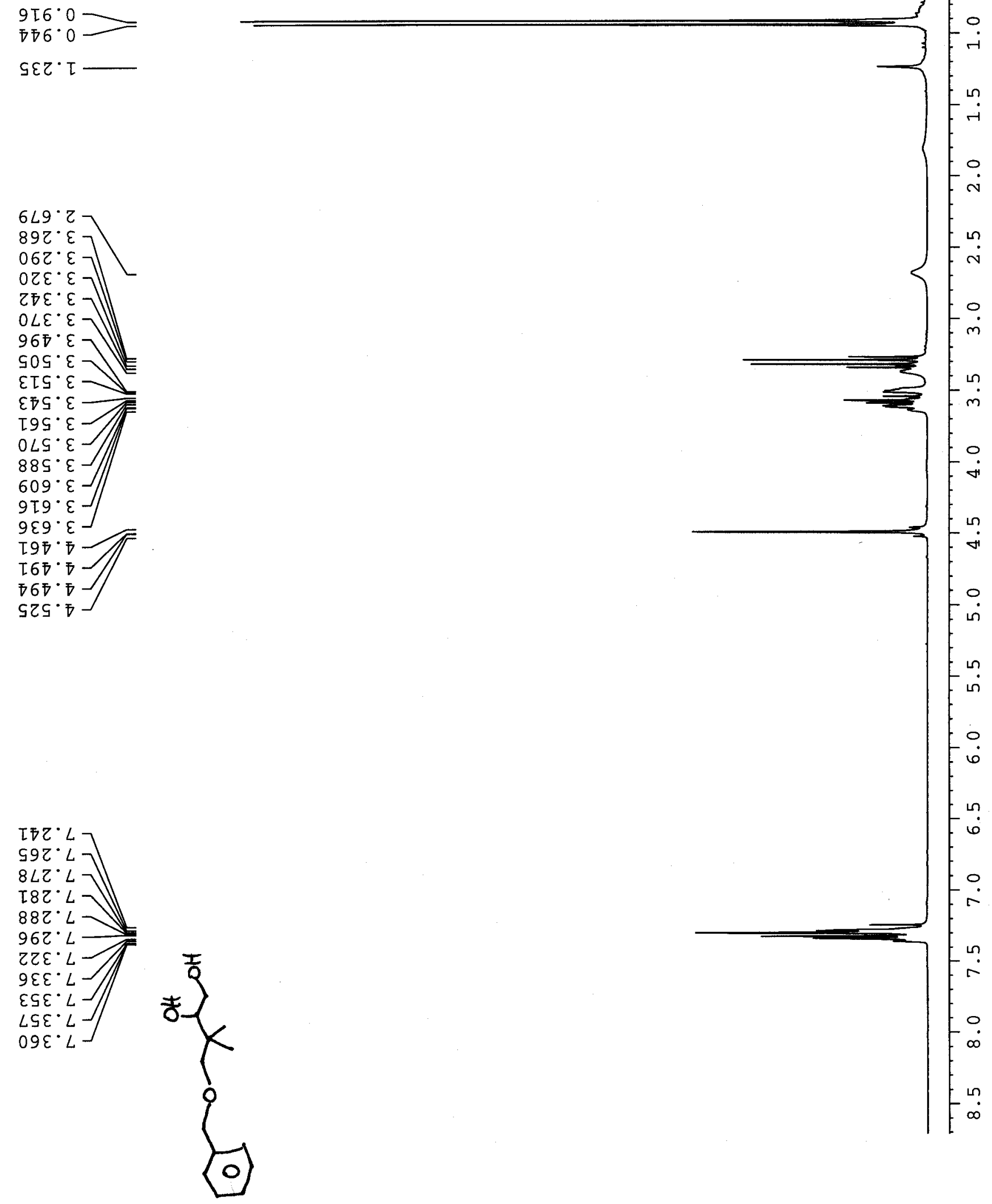

아 

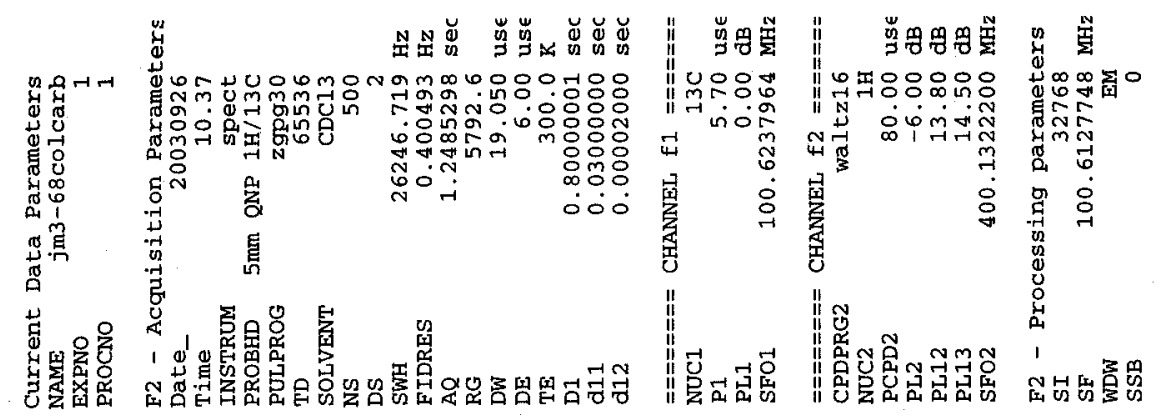

$229^{\circ} 02$

$990^{\circ} \varepsilon 乙$

$\varepsilon 99^{\circ} 6 Z$

$\bar{\nabla} \bar{\nabla}\llcorner\varepsilon$

$\tau \angle 8^{\circ} 29$

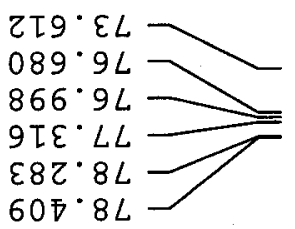

$\sum_{0}^{ \pm}$

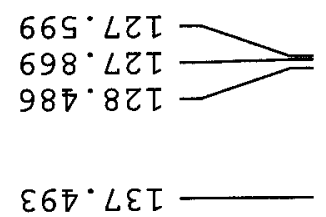

$\varepsilon 6 \nabla^{\circ}\llcorner\varepsilon \tau$

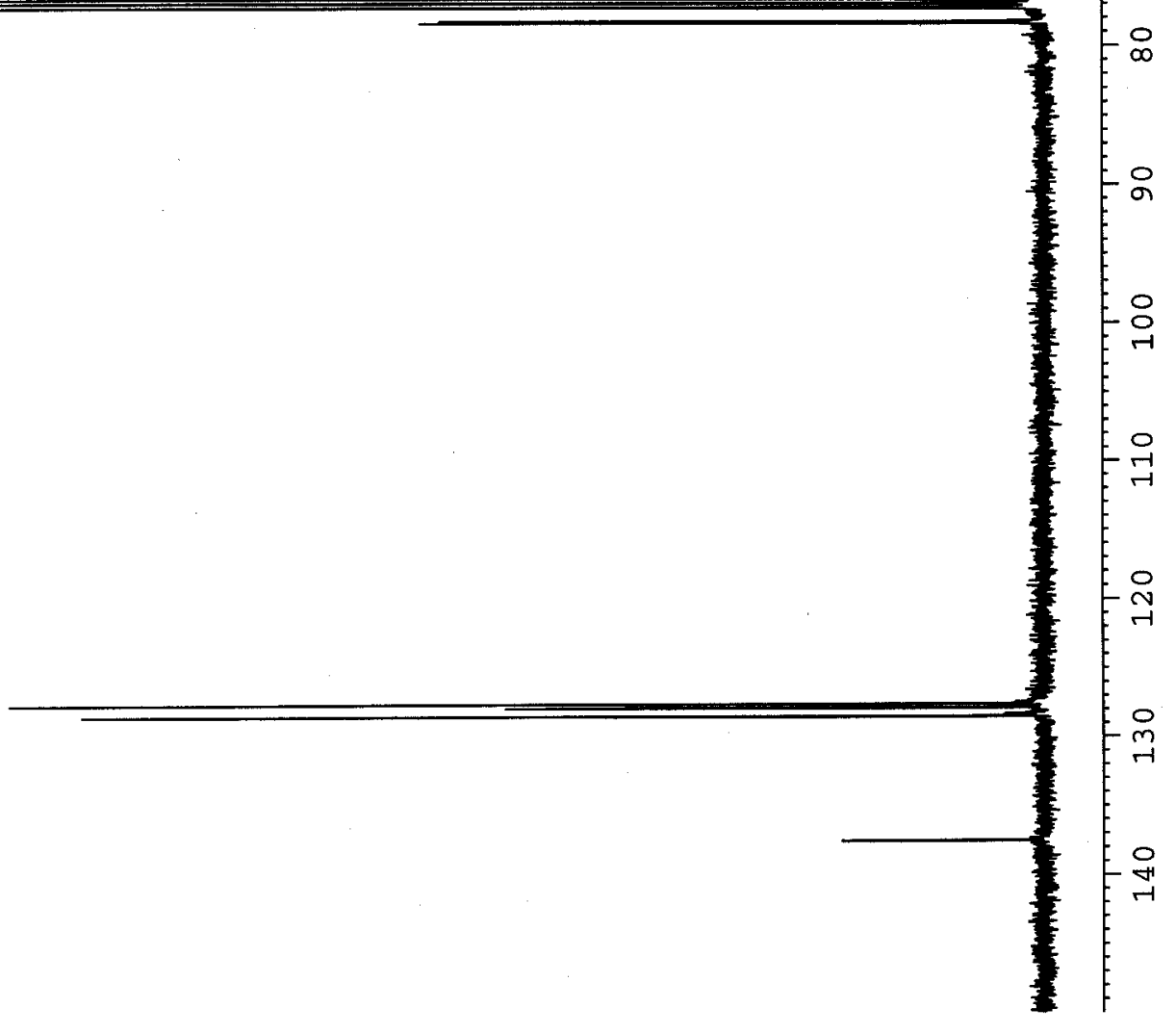

Page 23 


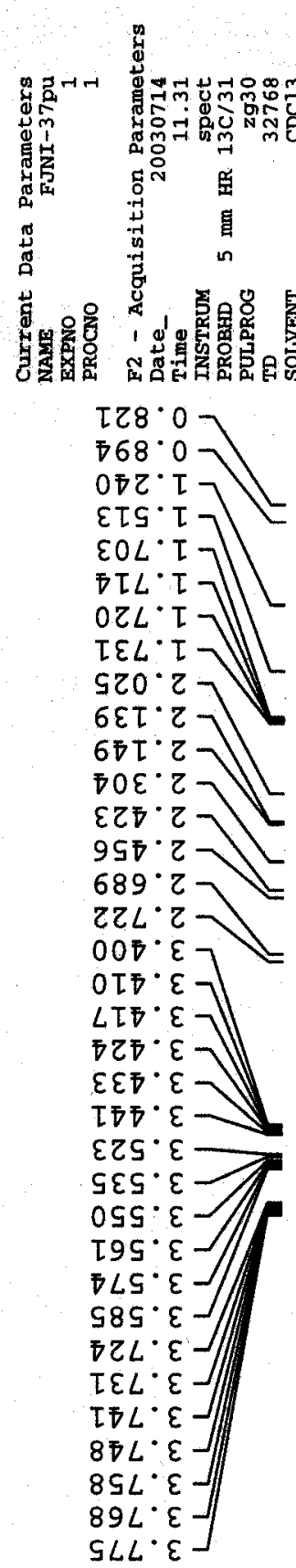

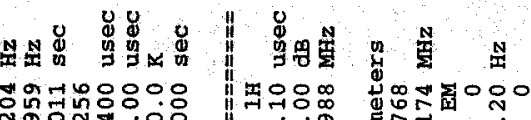

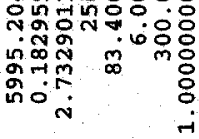

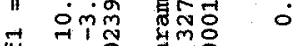

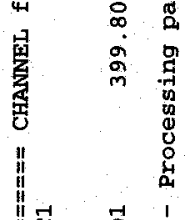

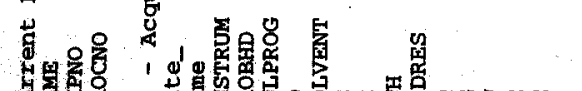

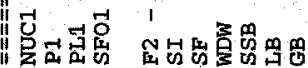
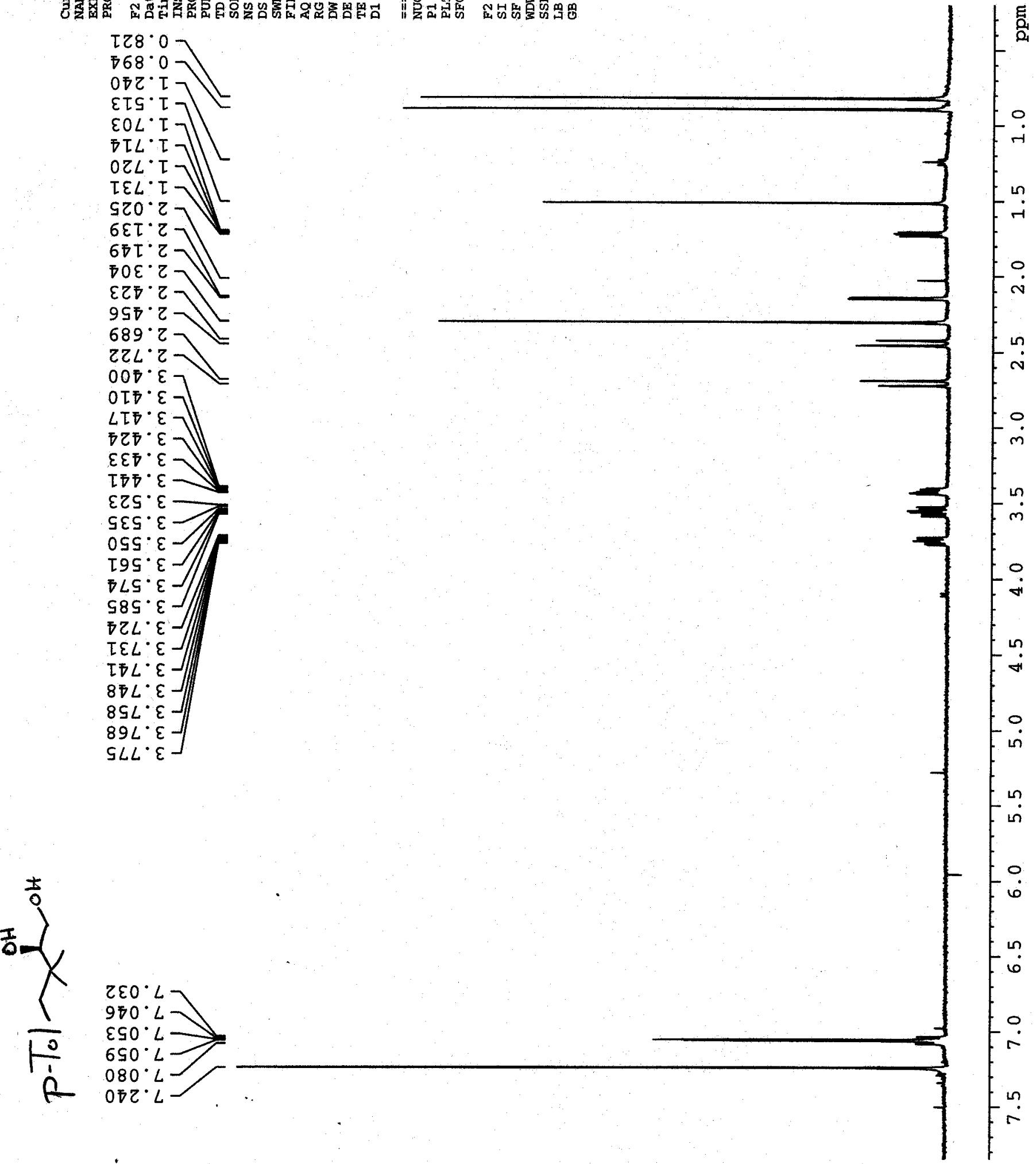


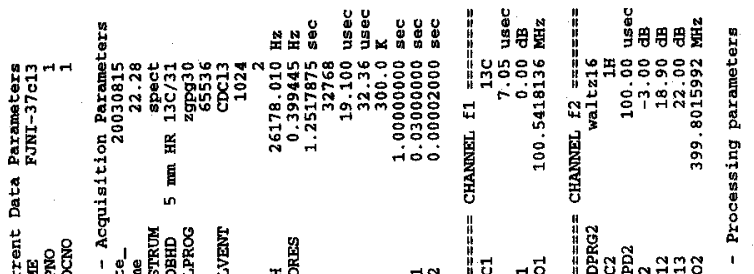

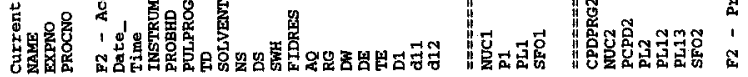

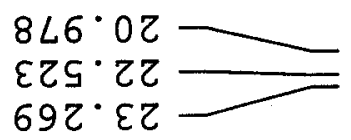

$L L \varepsilon^{\circ} L \varepsilon$

$66 L^{\circ}$ D

$\tau 乙 \tau \cdot \varepsilon 9$

$189^{\circ} 9 L$

$866^{\circ} 9 L$

9 IE $L L$

$\angle 89^{\circ} L L$
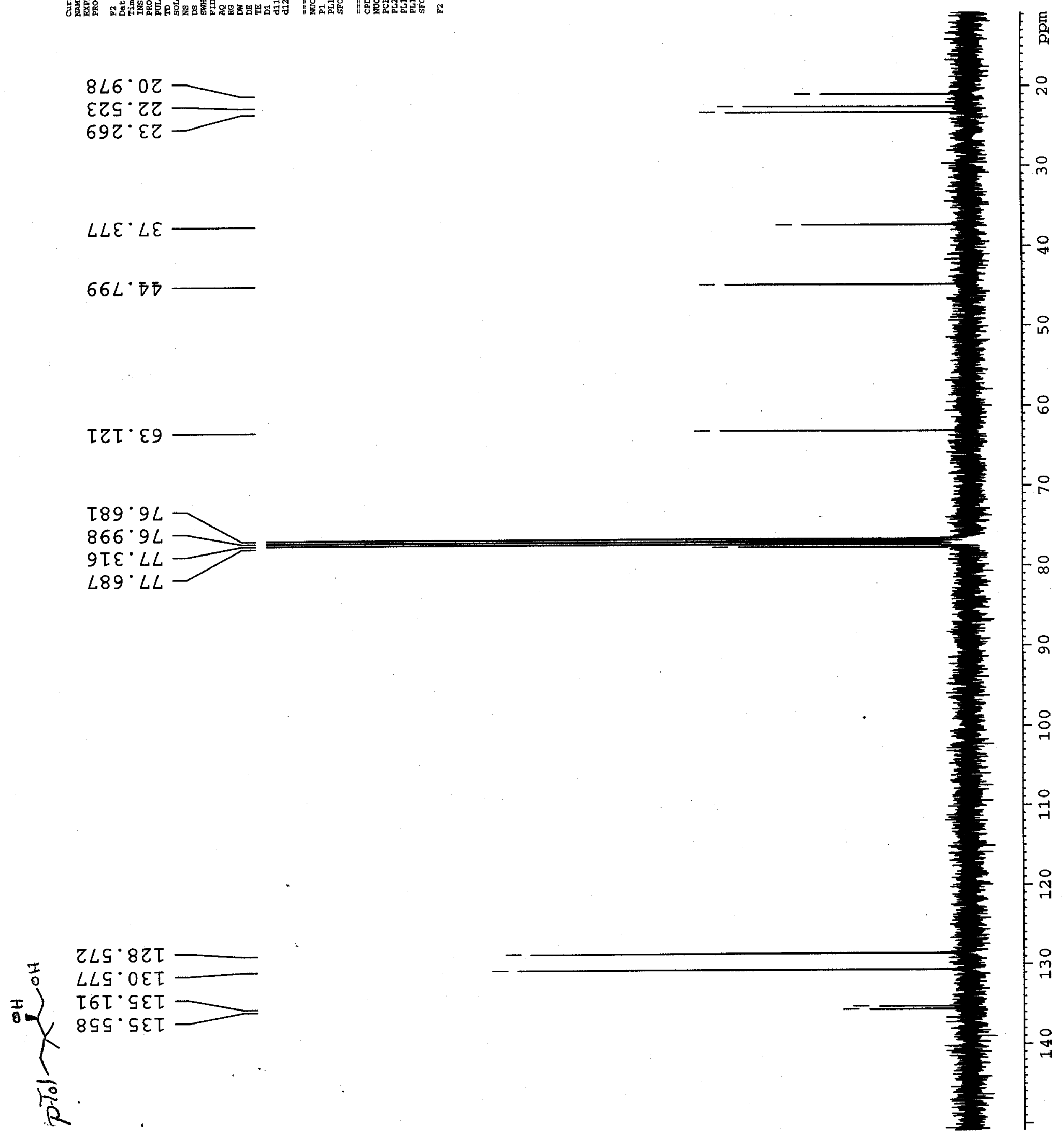

Page 25 


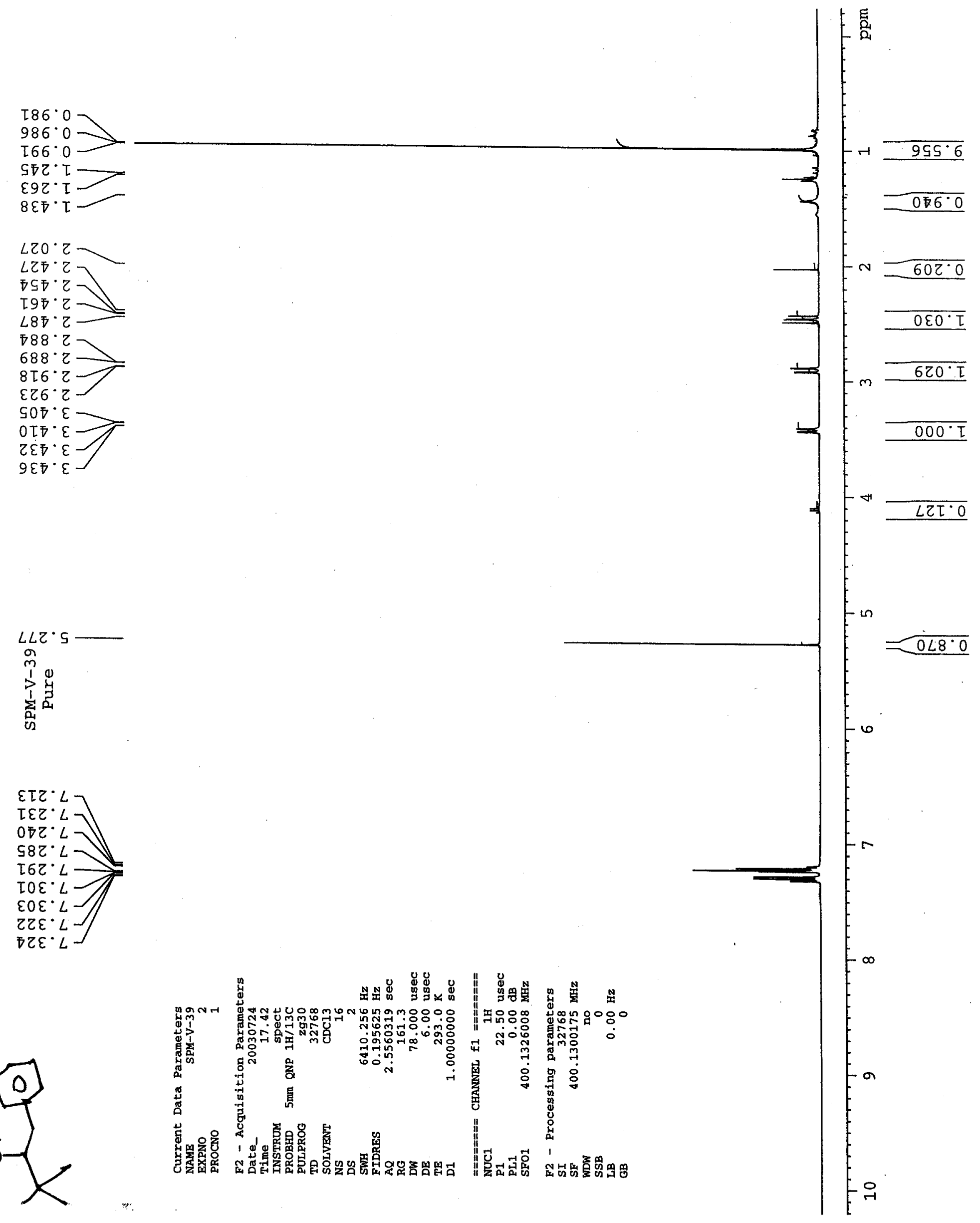

Page 26 


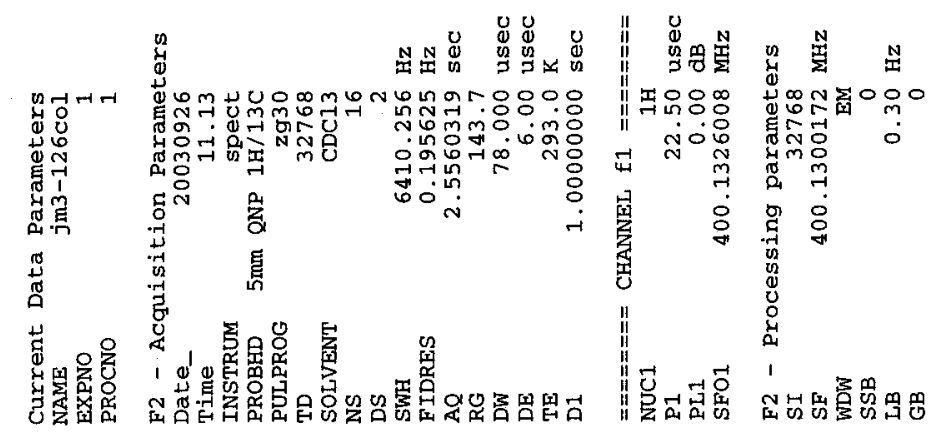

$586^{\circ} 0$

$005^{\circ} \mathrm{T}$

$000^{\circ} Z$

$\angle Z D^{\circ} \mathrm{Z}$

$\varepsilon \varepsilon \bar{\nabla} \cdot$

095 $\mathrm{Z}$

$\angle S 8^{\circ} Z$

$\mathrm{L68} \cdot \mathrm{Z}=$

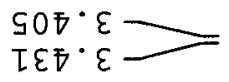

$68 L \cdot \varepsilon$
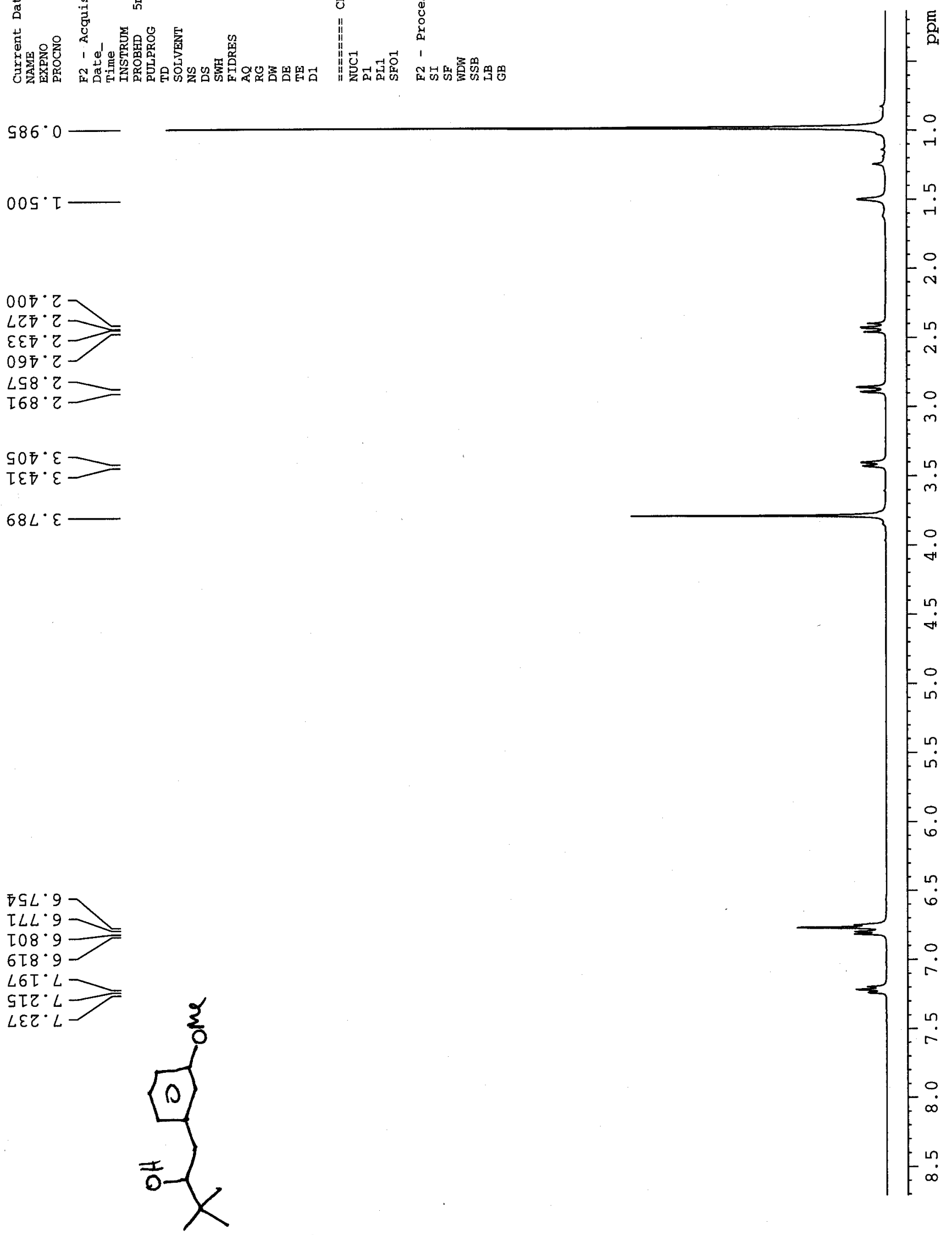


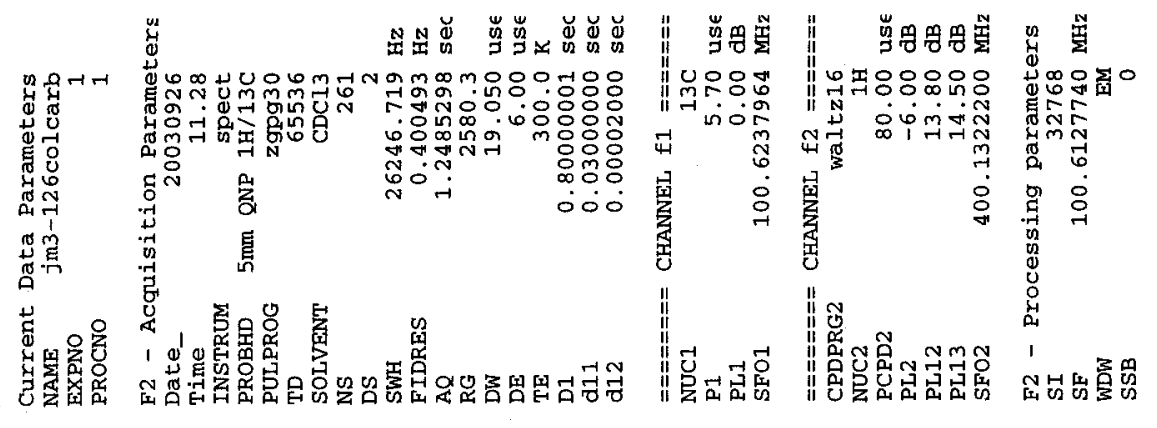

$\angle \varepsilon 8^{\circ} \varsigma \mathrm{Z}$

$\varepsilon L L^{\circ} \bar{\nabla}$

Eऽฐ $8 \varepsilon$

$8 \varepsilon \tau \cdot \varsigma \varsigma$

$589^{\circ} 9$
$Z 00^{\circ} L$

OZE $L L$

ร97.08

ZSL.III

โ8 $8^{\circ}$ 五工

$265^{\circ} \tau Z \tau$

$8 \varepsilon S \cdot 6 \tau I$

$00 S^{*} \operatorname{tzI}$
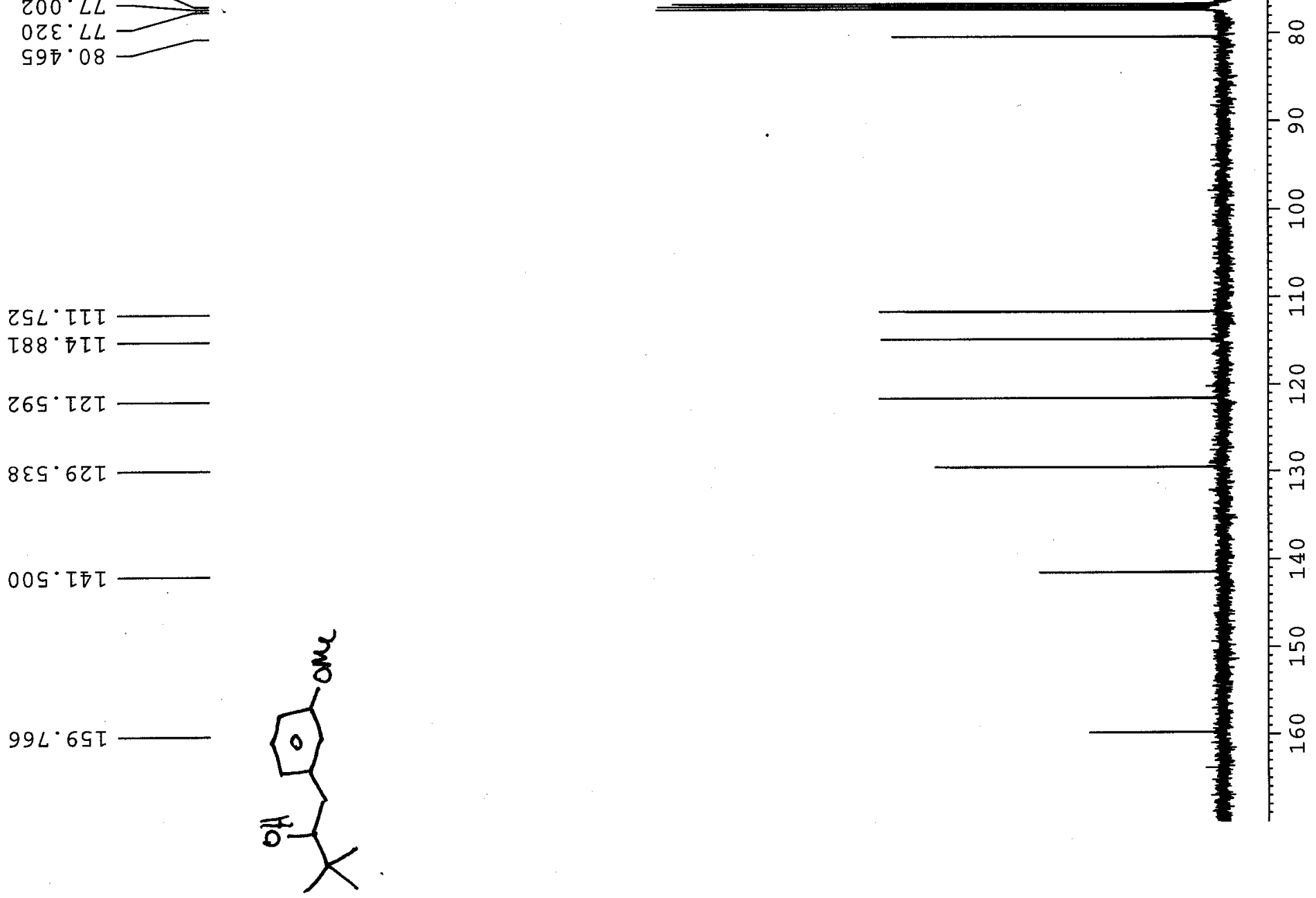

Page 28 

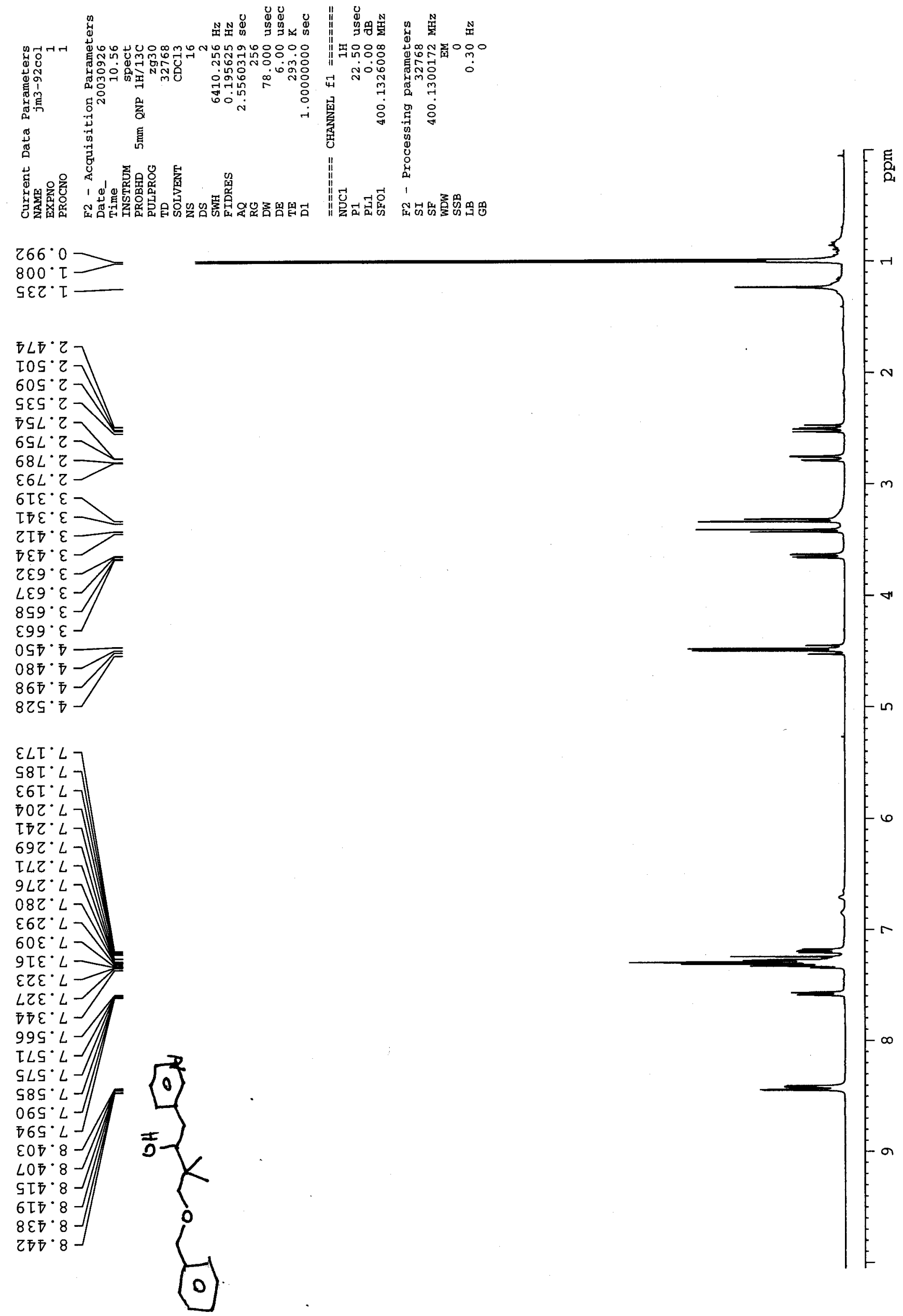

Page 29 


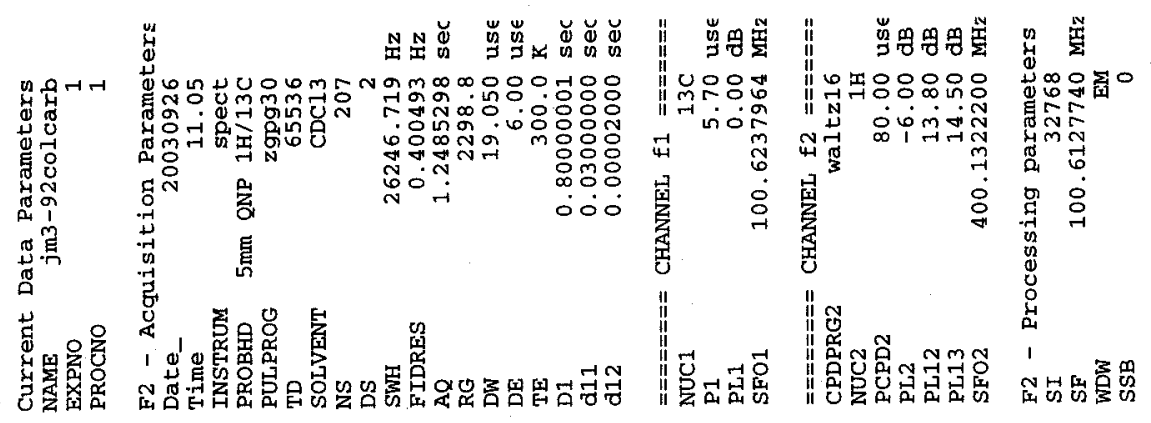

$6 \angle 9^{\circ} 6 \mathrm{I}$

$58 L \cdot 27$

$Z \angle 9^{\circ} 6 Z$

ऽฐ๐ $\subsetneq \varepsilon$

$6 \varepsilon \varsigma^{\cdot} 8 \varepsilon$

$6 \varepsilon 9^{\circ} \varepsilon L$ $\varepsilon 89^{\circ} 9 L$

T00 $\mathrm{LL}$ $6 \tau \varepsilon^{\circ} L L$

$6 \varepsilon 0^{\circ} 6 L$

9๊5 $6 L$ $\varepsilon \subseteq \tau \cdot \varepsilon \tau \tau$

T09. $L Z \tau$

$608^{\circ} \angle Z T$

乙6L $\varsigma \varepsilon \tau$

ZTO $L E T$

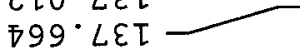

$\angle 9 Z^{\circ} \angle D I$

万9D. OST

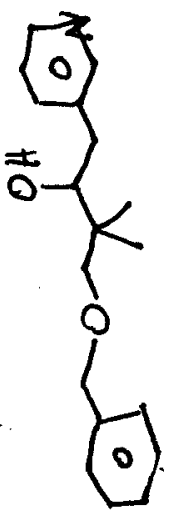

-)

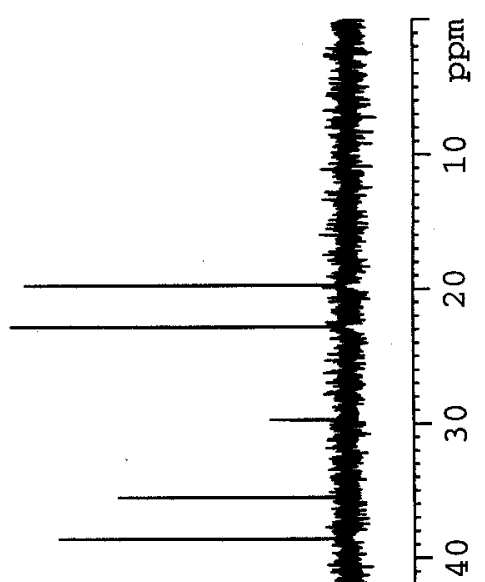

in

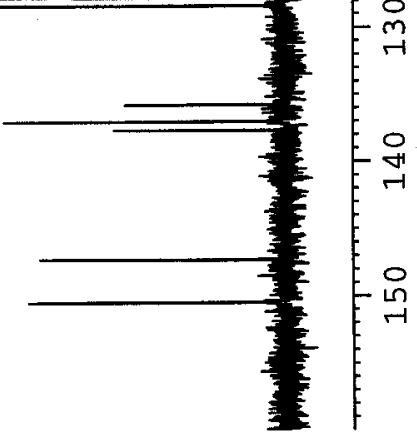



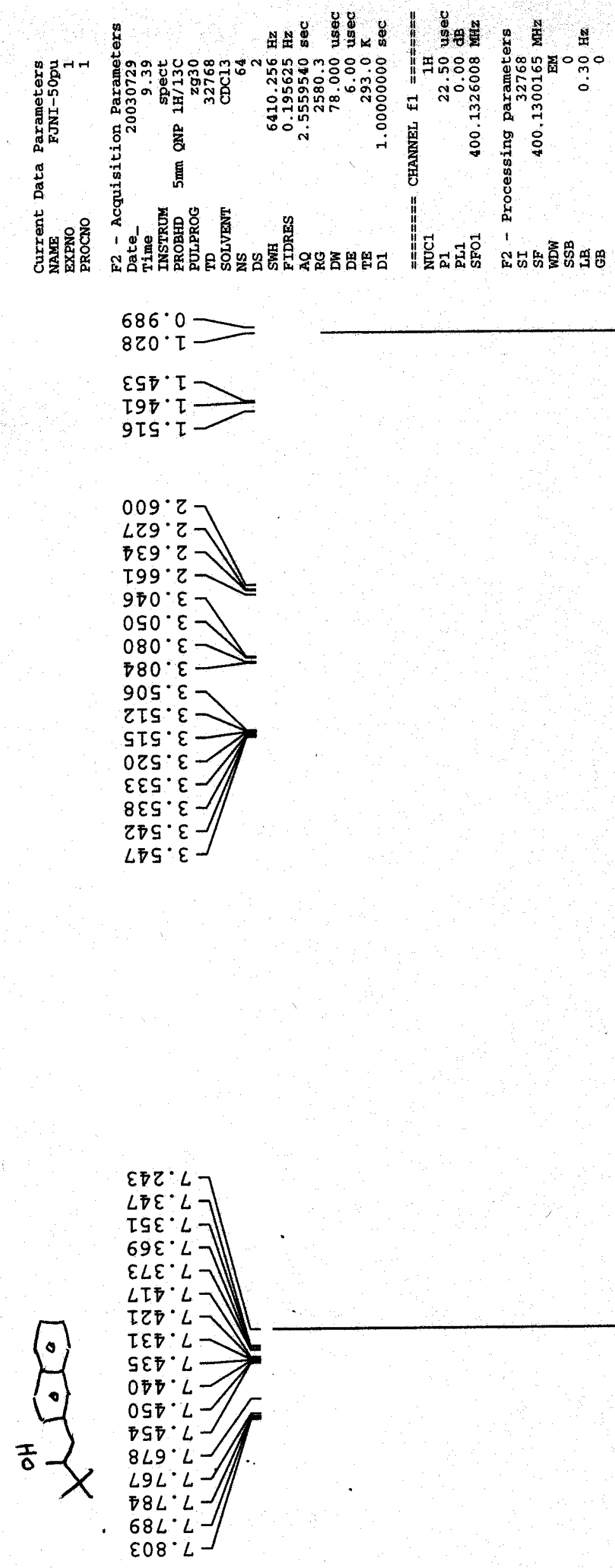

Page 31 


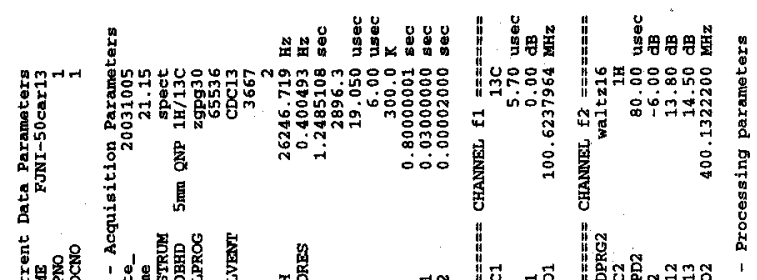

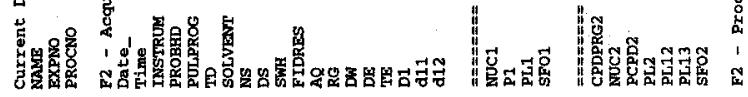

$8 Z \varepsilon \cdot 9 \tau$

$0 โ \varepsilon \cdot \varsigma \varepsilon$

$786^{\circ} 8 \varepsilon$

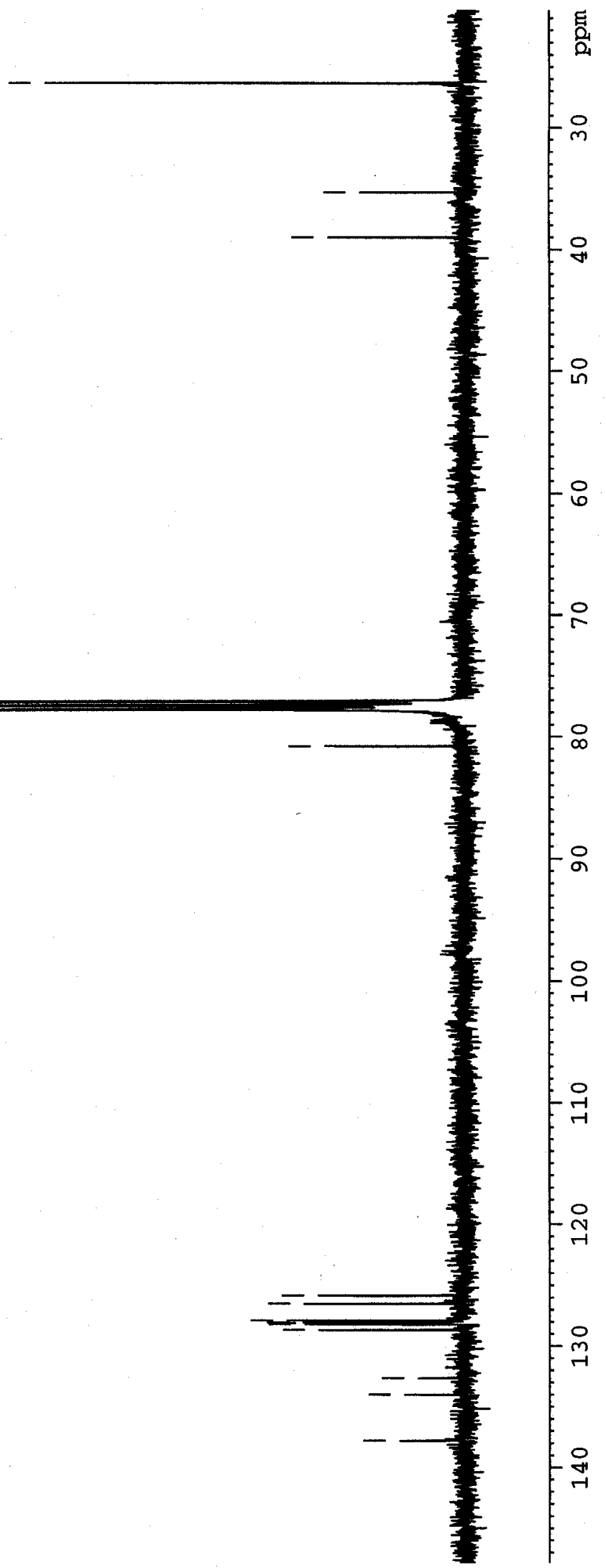

Page 32 


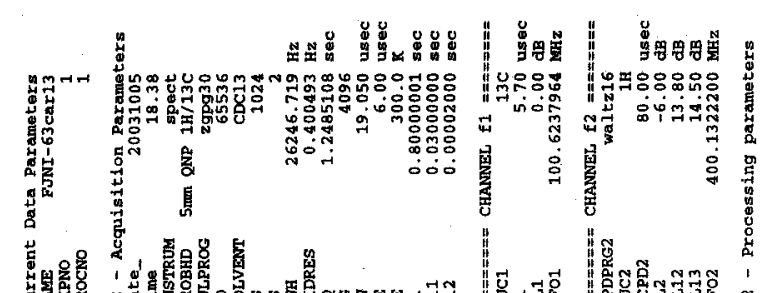

$766^{\circ} \mathrm{g} 2$

9โS $9 \varepsilon$

$\varsigma 8 \nabla^{\circ} 8 \varepsilon$

$$
\text { 政 }
$$
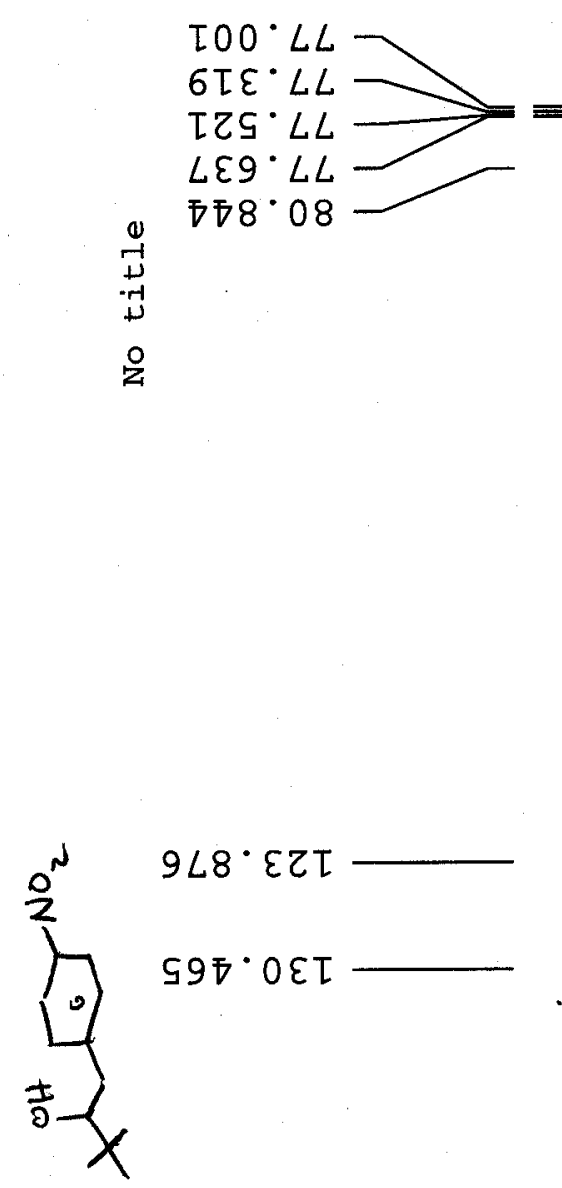

$668^{\circ} \cdot 9 \bar{T}$

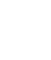

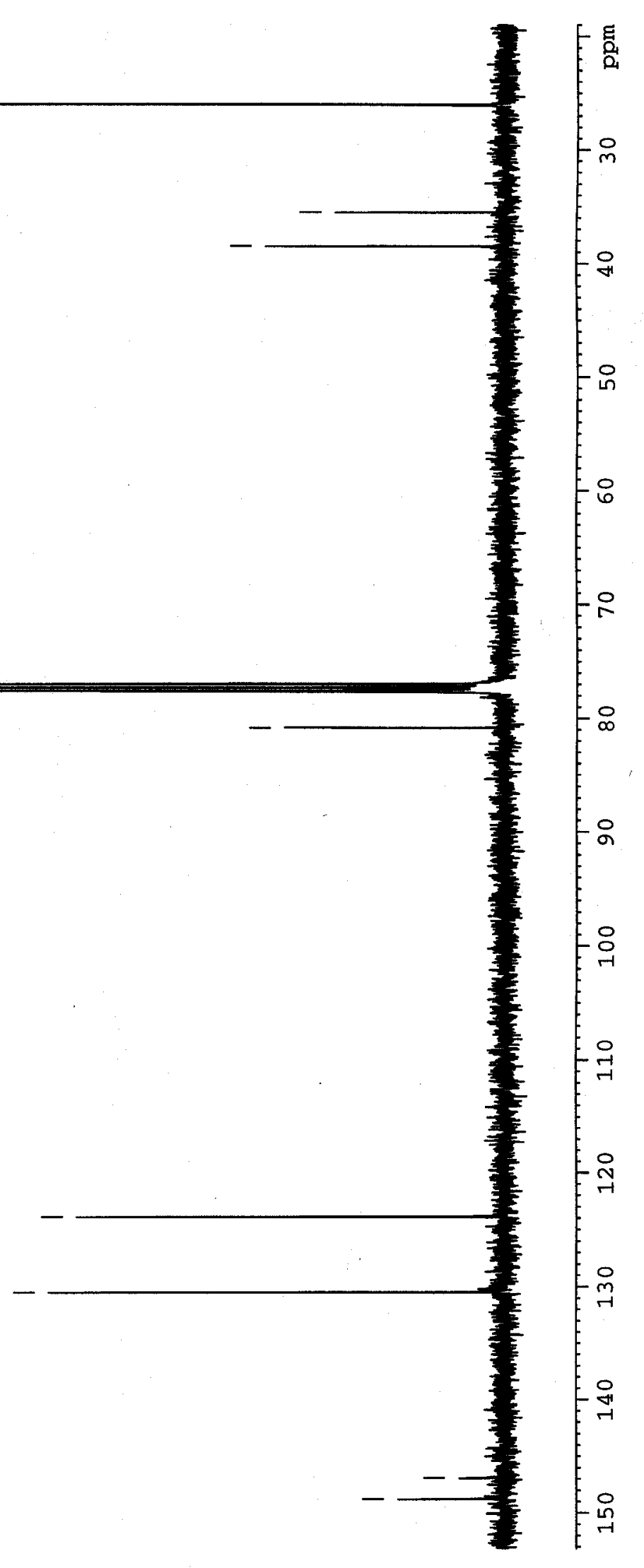




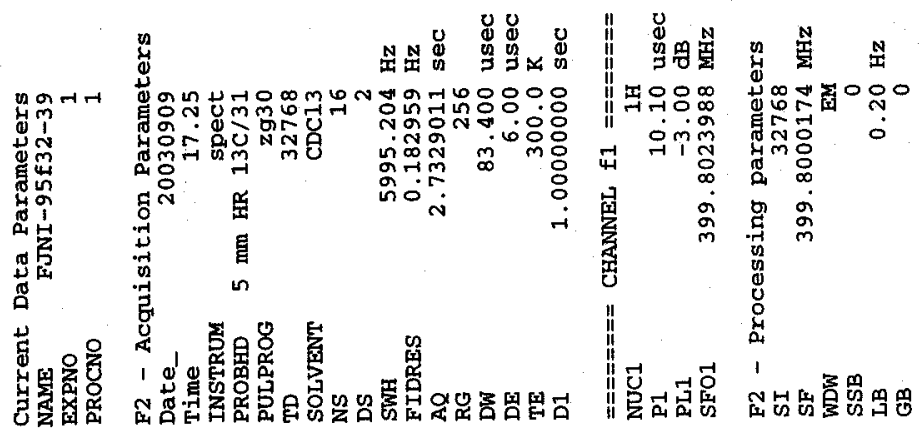
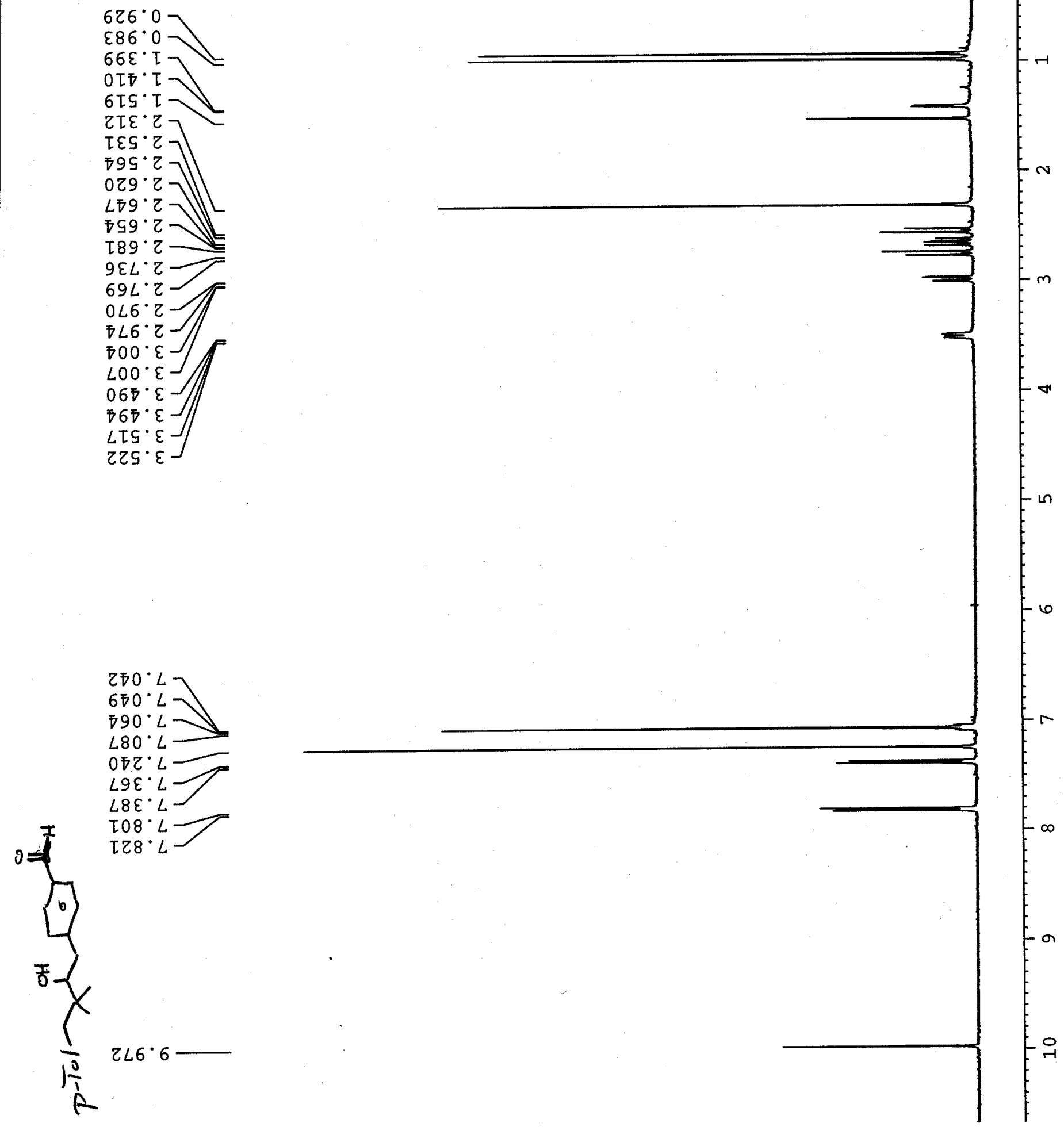


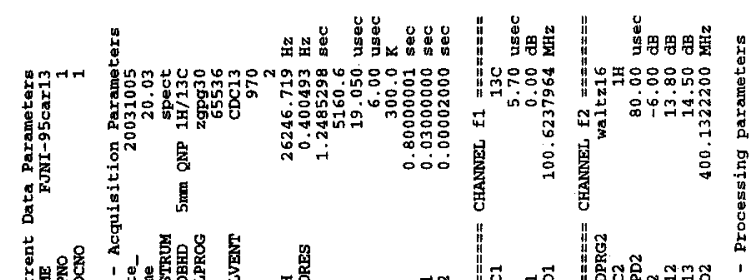

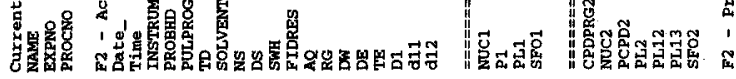

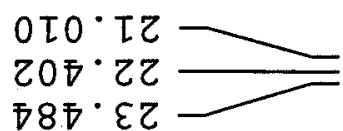

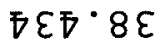

$8 \varepsilon 8 \cdot 8 \varepsilon$

$\varsigma \varepsilon^{*} \bar{~}$

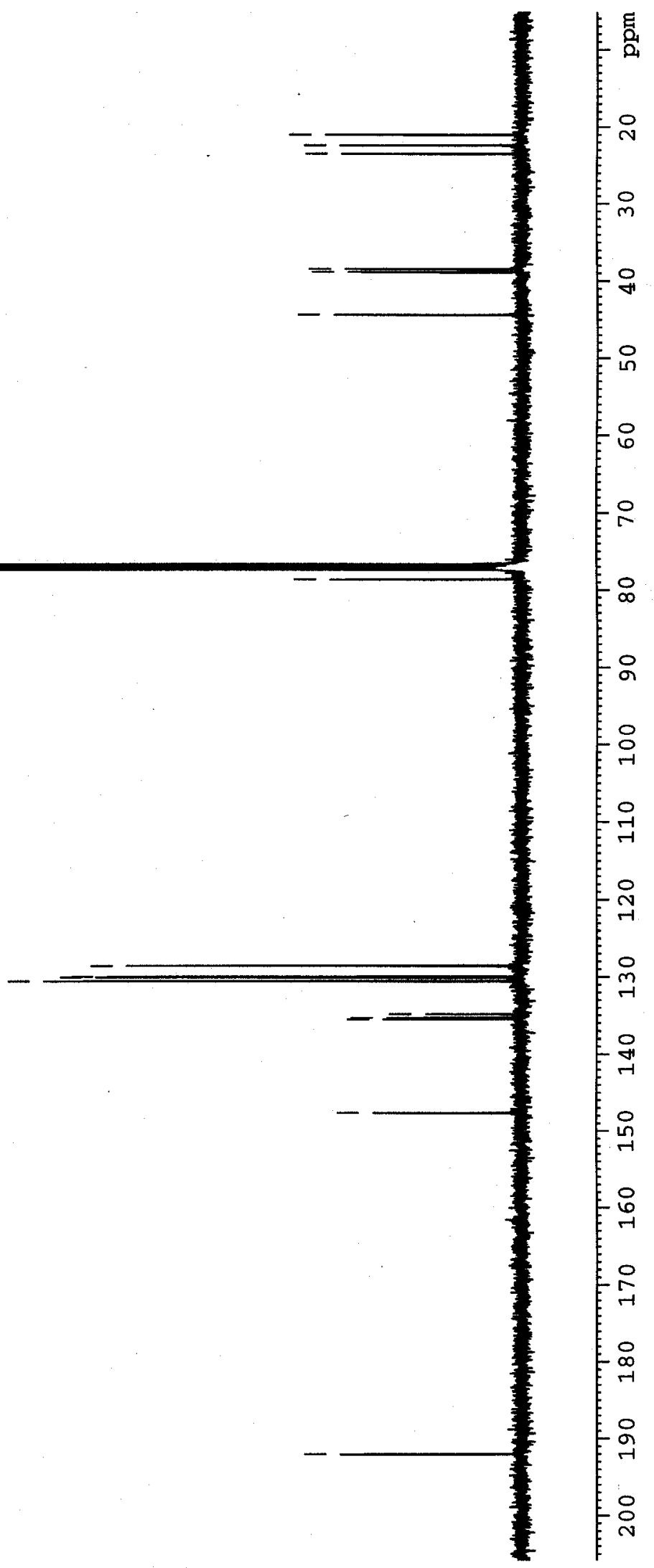



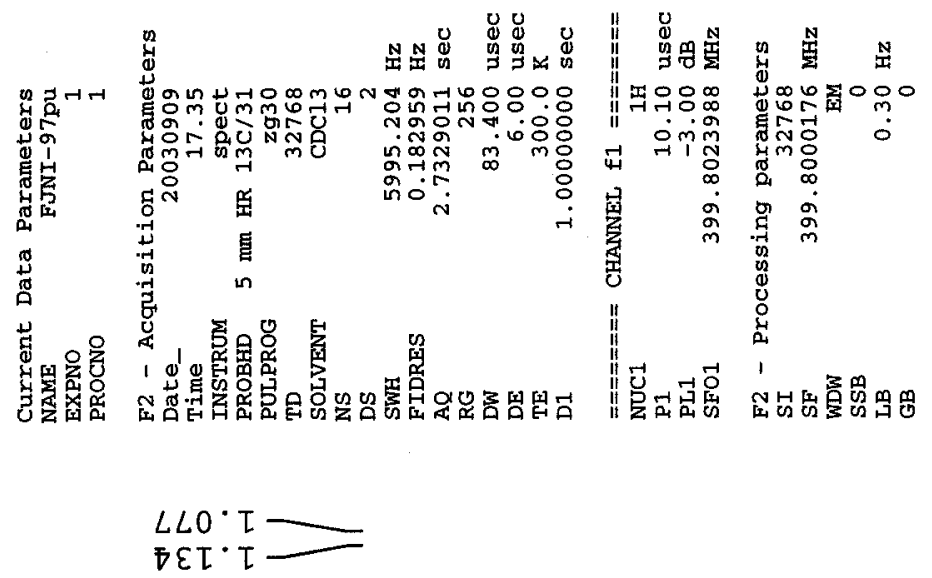

$\varepsilon \mathrm{ZL} \cdot \mathrm{T}$

$\angle S Z$

$799 \cdot z$

$\angle 8 L \cdot Z]$

$278^{\circ} z$

$\angle 28$

$\varsigma \varepsilon 8^{\circ} \mathrm{Z}$

$\nabla 98^{\circ} \mathrm{Z}$

$600^{\circ} \varepsilon$

Ђ๐० $\varepsilon$

Sโ $\varepsilon-$

$96 \tau \cdot \varepsilon$

$00 \tau \cdot \varepsilon]$

$\angle 08^{\circ}$

$\varsigma \varepsilon 8 \cdot \varepsilon$
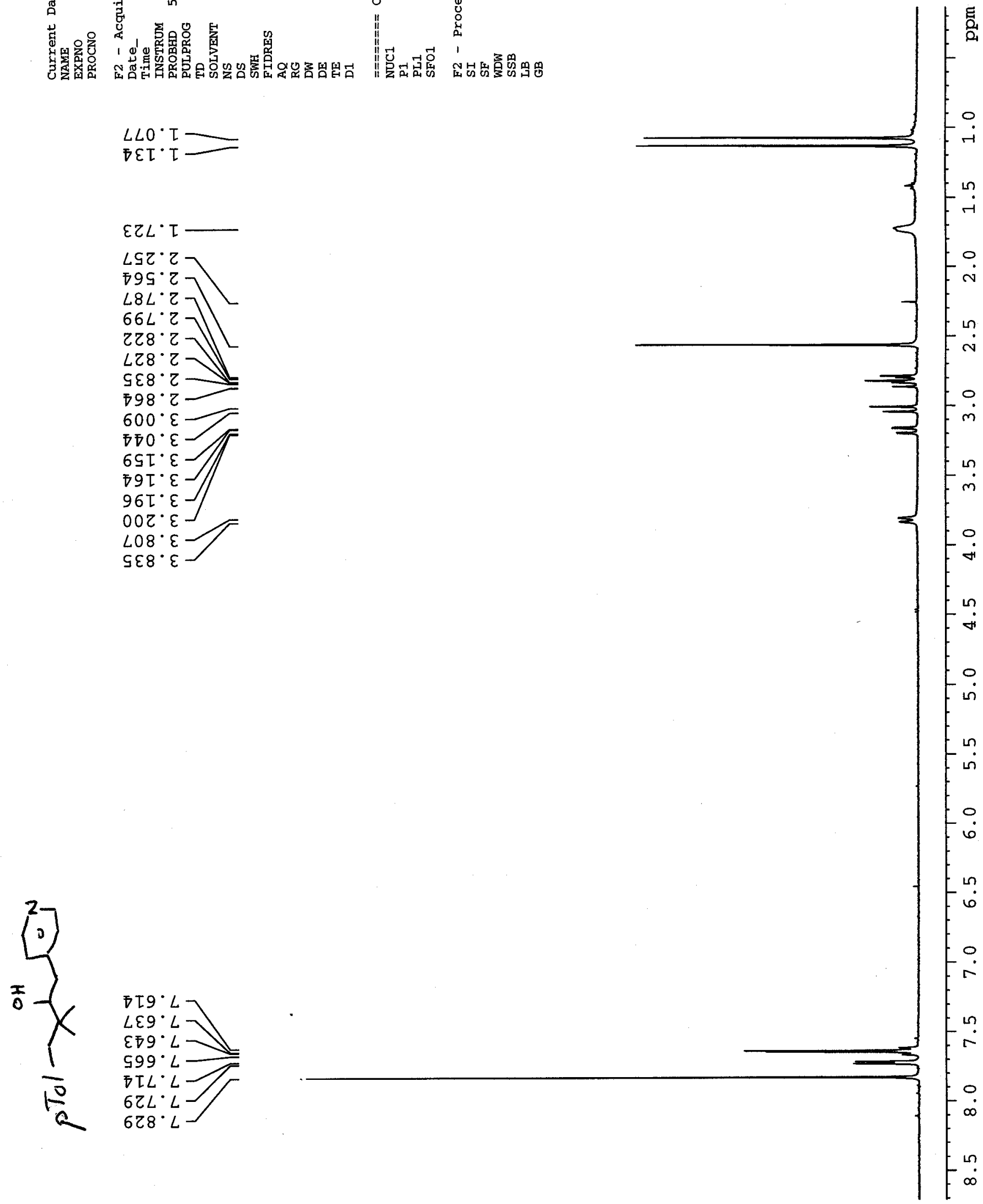

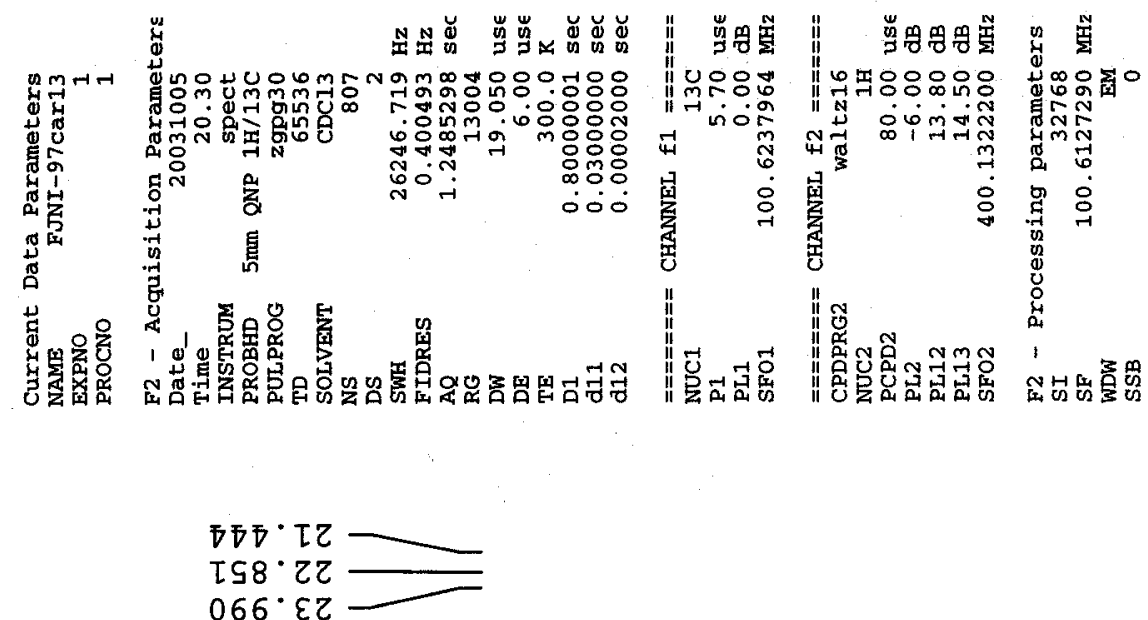

$066^{\circ} \varepsilon z$

โST. $8 \varepsilon$

$0 โ \varepsilon \cdot 6 \varepsilon$

てะ8 Ð๐

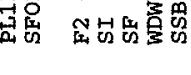

$Z Z T \cdot L L$

$8 S L \cdot L L$

$07 \overline{0} \cdot 8 L$
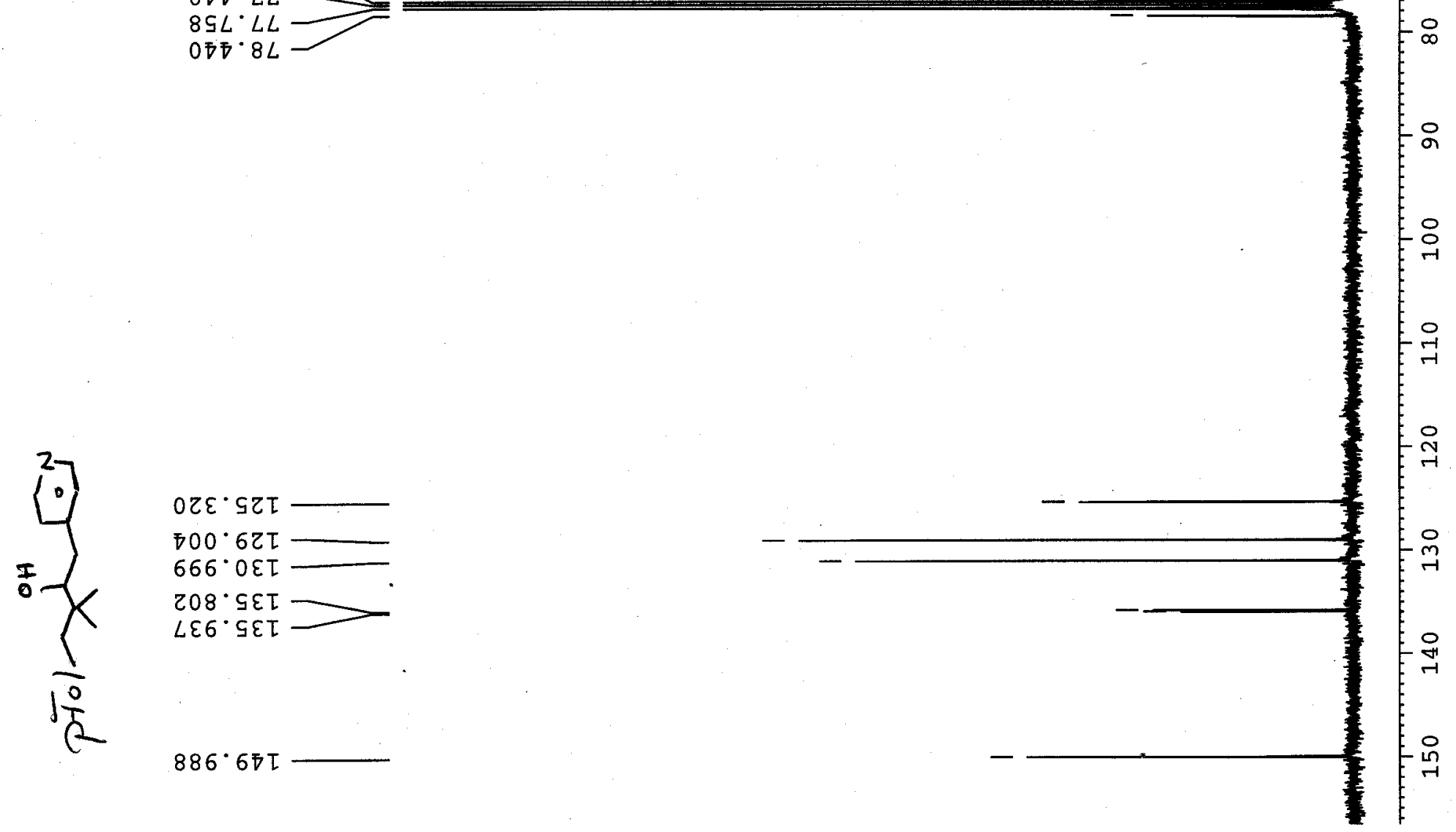

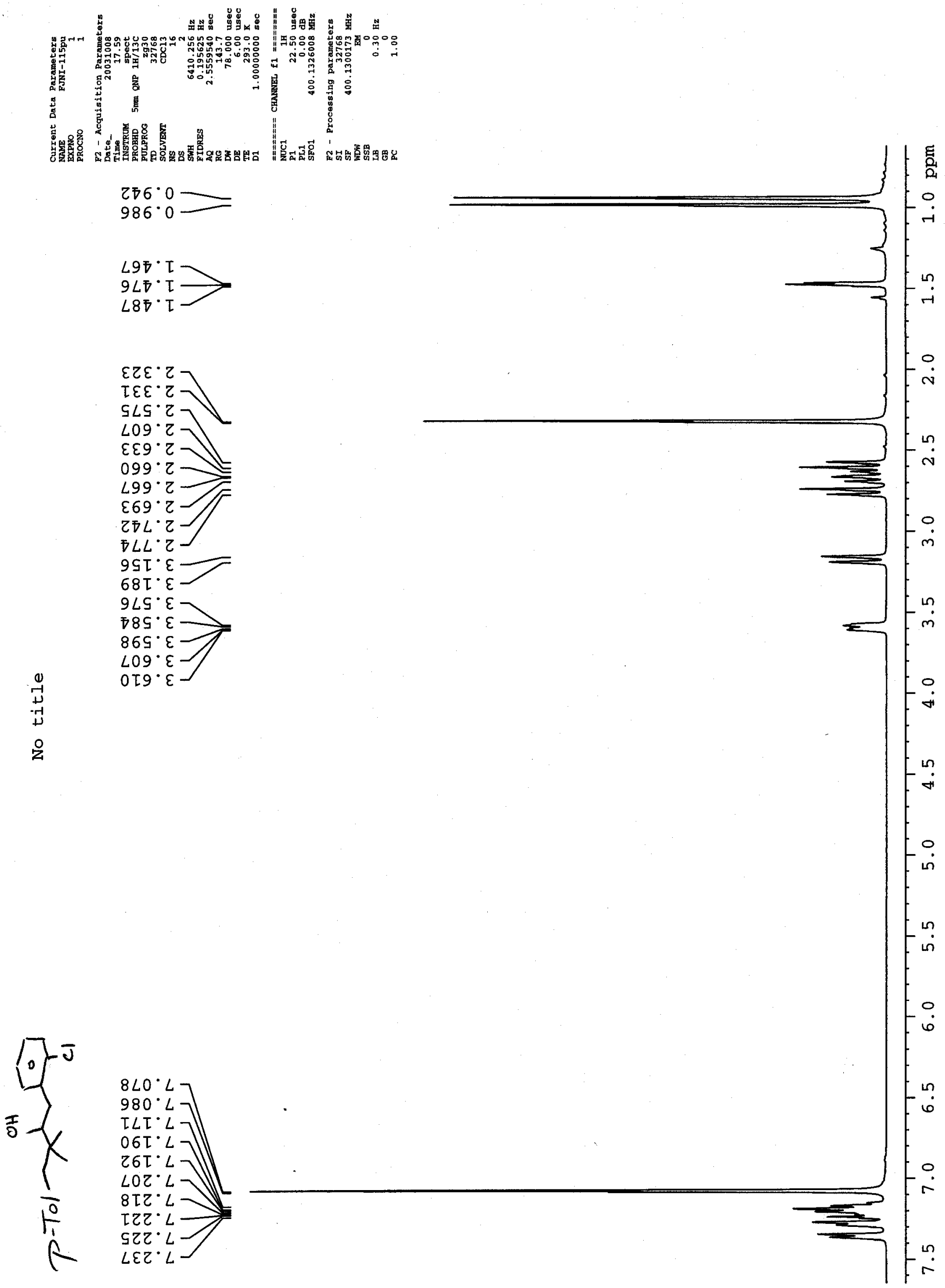

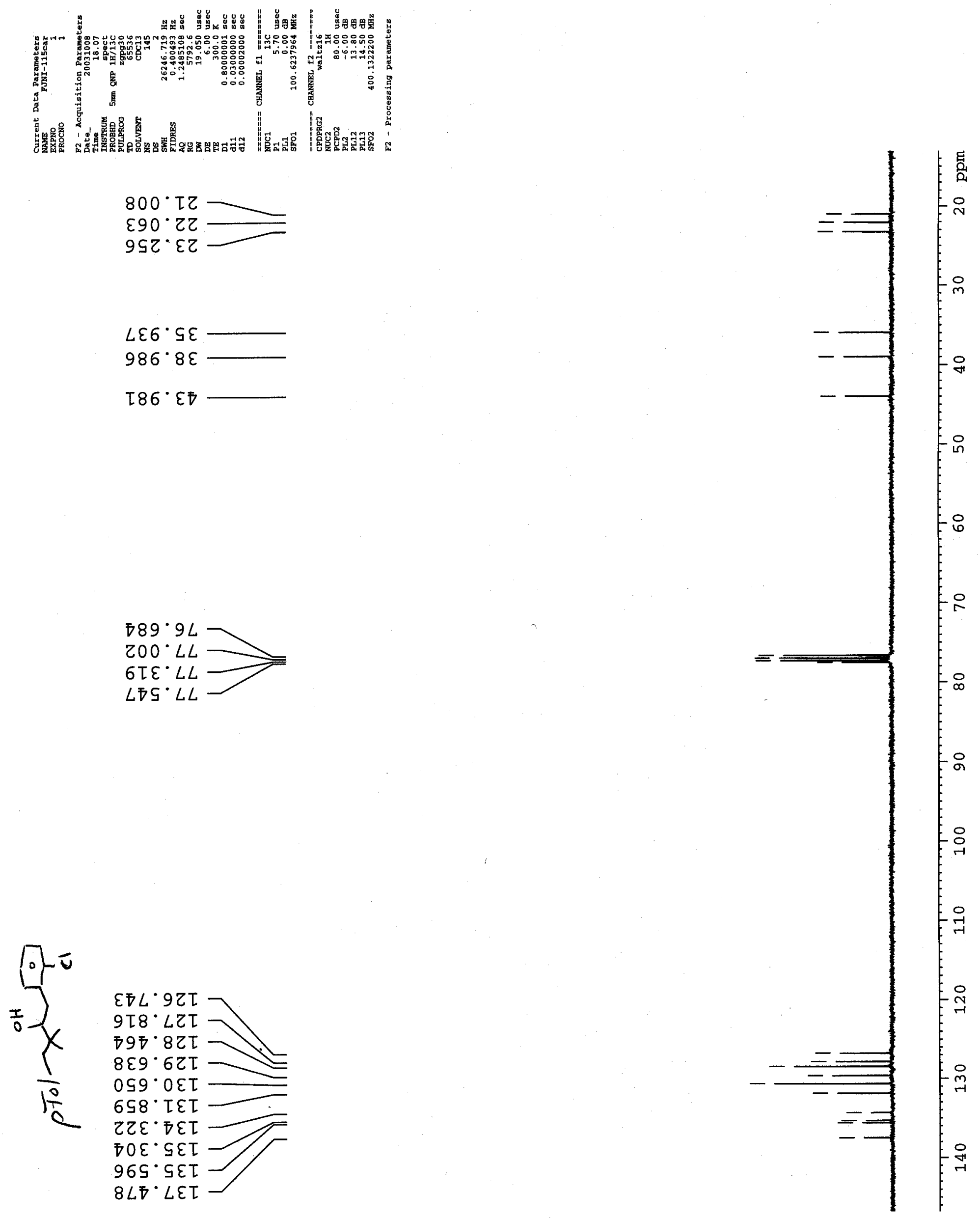

Page 40 

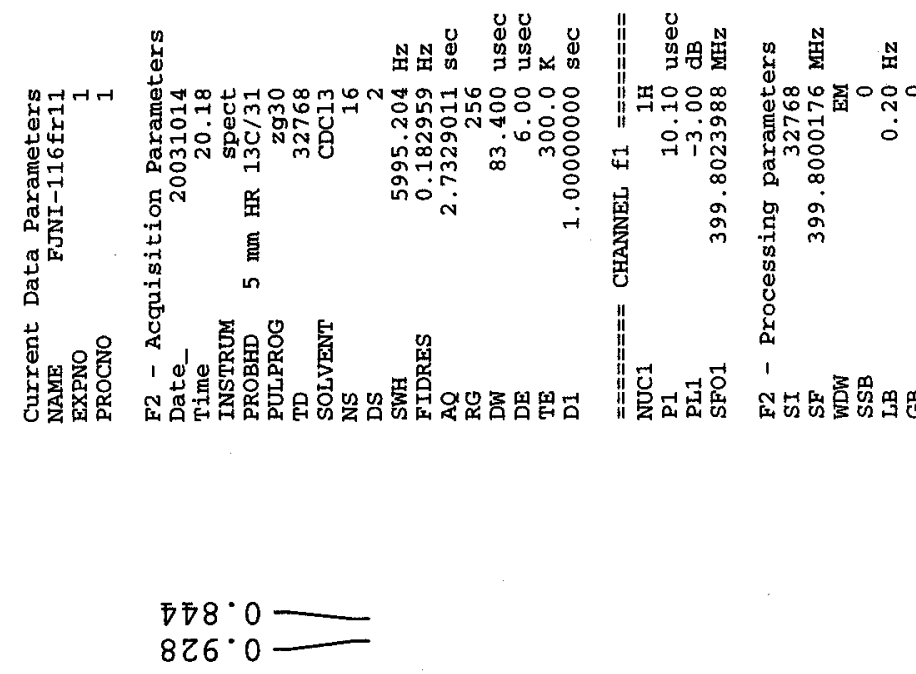

$\varepsilon \tau \varepsilon^{\circ}$

6IS $\tau$
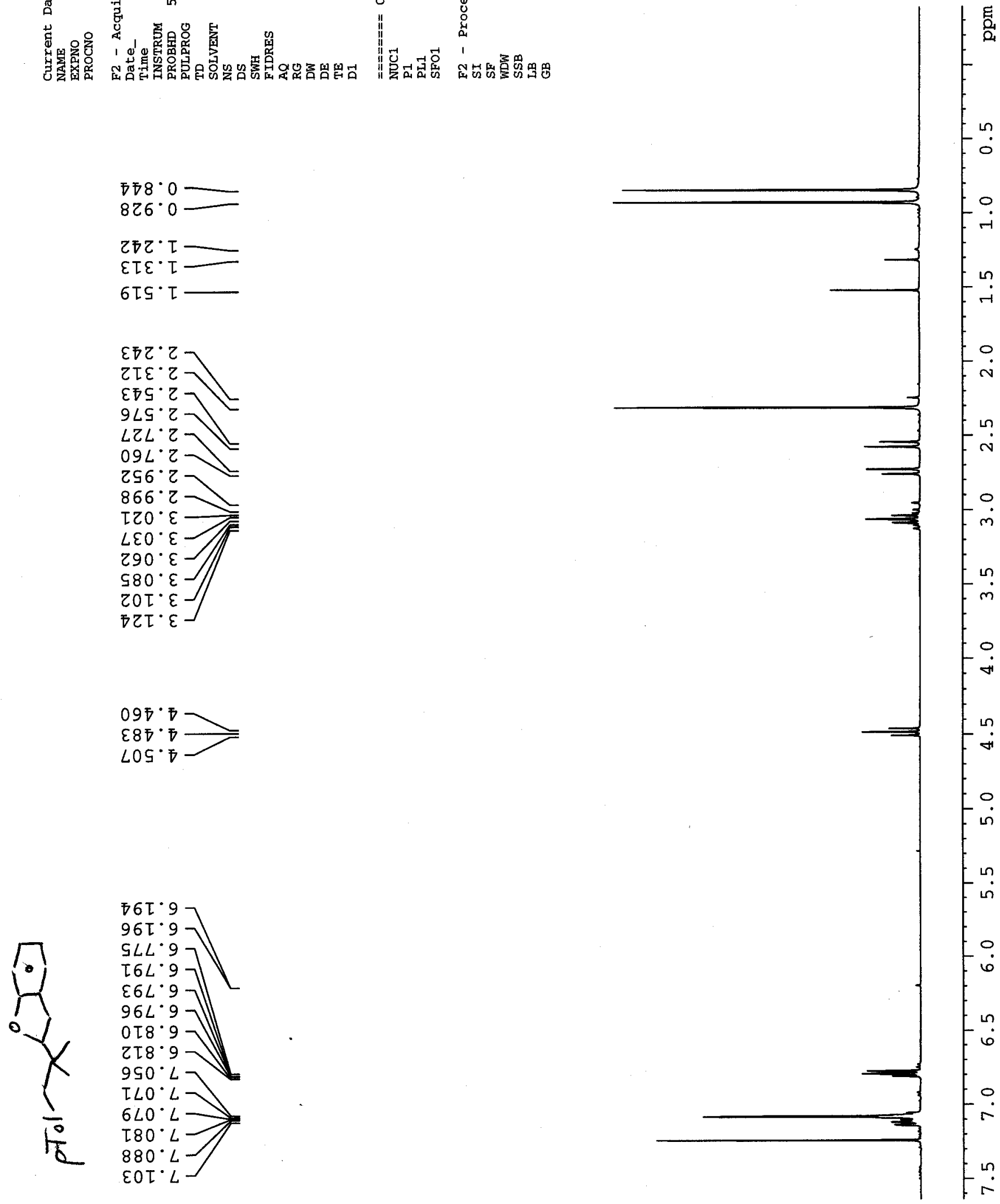

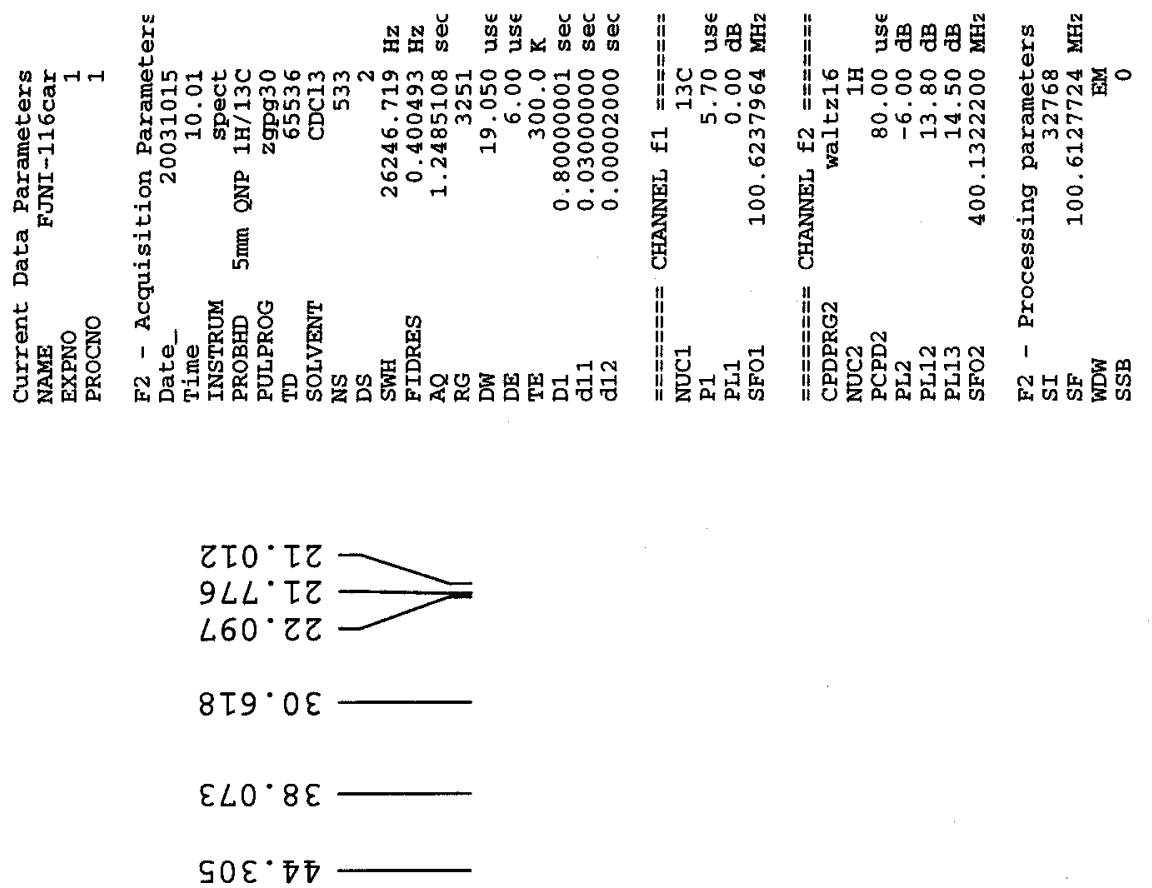

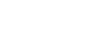

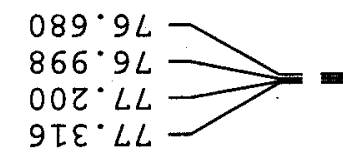

โร9・88
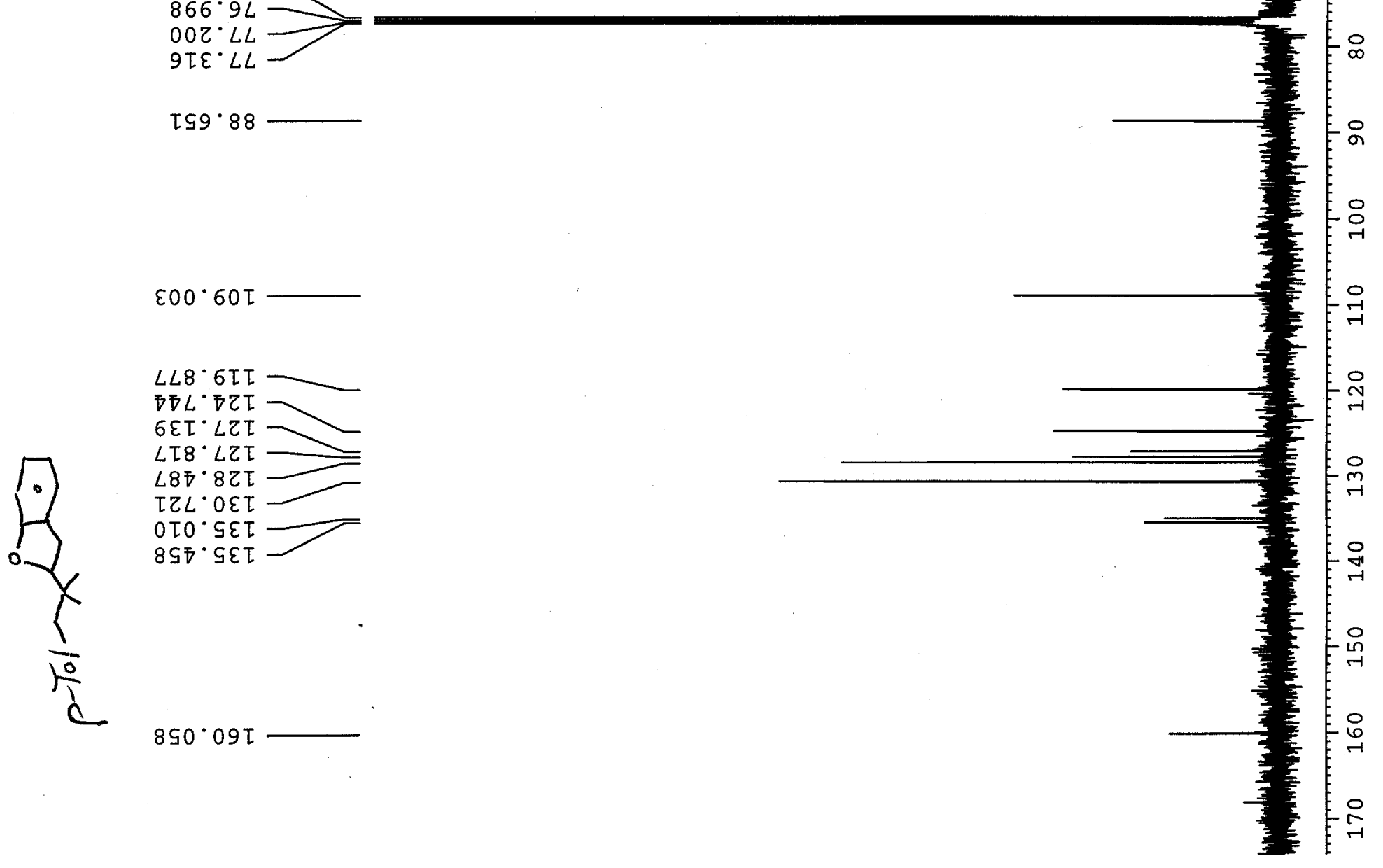

Page 42 